\title{
1 A sustained type I IFN-neutrophil-IL-18 axis drives pathology during mucosal viral infection
}

3 Tania J. Lebratti ${ }^{1,}, \#$, Ying Shiang Lim ${ }^{1, \#}$, Adjoa Cofie ${ }^{1}$, Prabhakar S. Andey ${ }^{2}$, Xiaoping Jiang ${ }^{1}$,

4 Jason M. Scott ${ }^{1}$, Maria Rita Fabbrizi ${ }^{1,}$, , Ayse N. Ozanturk ${ }^{1}$, Christine T.N. Pham ${ }^{4}$, Regina A.

5 Clemens $^{3}$, Maxim Artyomov², Mary C. Dinauer ${ }^{5}$, Haina Shin ${ }^{1, *}$

$7{ }^{1}$ Department of Medicine/Division of Infectious Diseases; ${ }^{2}$ Department of Pathology and

8 Immunology; ${ }^{3}$ Department of Pediatrics/Division of Critical Care Medicine; ${ }^{4}$ Department of

9 Medicine/Division of Rheumatology; ${ }^{5}$ Department of Pediatrics/Hematology and Oncology,

10 Washington University School of Medicine, St Louis, MO, USA

11

$12{ }^{\S}$ Current address: Bayer, Crop Science Division, St Louis, MO, USA

13 Current address: Department of Molecular and Clinical Cancer Medicine, Northwest Cancer

14 Research Centre, University of Liverpool, Liverpool, UK

15

$16{ }^{\#}$ These authors contributed equally.

$17 \quad$ * Corresponding author: haina.shin@wustl.edu

18

19 Running title: Pathogenic neutrophil responses during HSV-2 infection 


\section{ABSTRACT}

21 Neutrophil responses against pathogens must be balanced between protection and

22 immunopathology. Factors that determine these outcomes are not well-understood. In a mouse

23 model of genital herpes simplex virus-2 (HSV-2) infection, which results in severe genital

24 inflammation, antibody-mediated neutrophil depletion reduced disease. Comparative single cell

25 RNA-sequencing analysis of vaginal cells against a model of genital HSV-1 infection, which

26 results in mild inflammation, demonstrated sustained expression of interferon-stimulated genes

27 (ISGs) only after HSV-2 infection primarily within the neutrophil population. Both therapeutic

28 blockade of IFN $\alpha / \beta$ receptor 1 (IFNAR1) and genetic deletion of IFNAR1 in neutrophils

29 concomitantly decreased HSV-2 genital disease severity and vaginal IL-18 levels. Therapeutic

30 neutralization of IL-18 also diminished genital inflammation, indicating an important role for this

31 cytokine in promoting neutrophil-dependent immunopathology. Our study reveals that sustained

32 type I IFN signaling is a driver of pathogenic neutrophil responses, and identifies IL-18 as a novel

33 component of disease during genital HSV-2 infection. 


\section{INTRODUCTION}

Neutrophils are a critical component of the innate immune system. In humans, they are

37 the most abundant leukocyte in circulation and are often amongst the first wave of immune cells

38 responding to pathogen invasion. In the context of bacterial or fungal infection, including those

39 that are sexually transmitted, neutrophils are largely protective and can help eliminate pathogens

40 through a variety of effector functions, including phagocytosis, production of reactive oxygen

41 species (ROS), NET and protease release, and cytokine and chemokine secretion (Mayadas,

42 Cullere, \& Lowell, 2014; Pham, 2006; Tecchio, Micheletti, \& Cassatella, 2014). In contrast, the

43 role of neutrophils during viral infection is less clear (Galani \& Andreakos, 2015). While

44 neutrophils have been reported to neutralize several viruses and display protective qualities in vivo

45 (Akk, Springer, \& Pham, 2016; Craig N. Jenne et al., 2013; Saitoh et al., 2012; Tate et al., 2009;

46 Tate et al., 2011) they have also been associated with tissue damage, loss of viral control, and

47 increased mortality (Bai et al., 2010; Brandes, Klauschen, Kuchen, \& Germain, 2013; Kulkarni et

48 al., 2019; Narasaraju et al., 2011; Vidy et al., 2016).

Type I IFNs are potent regulators of neutrophil activity in a multitude of contexts. Type I

50 IFN can enhance recruitment of neutrophils to sites of infection, regulate neutrophil function and

51 drive immunopathology after infection by different classes pathogens, including Plasmodium spp.,

52 Candida spp. and Pseudomonas spp. (Majer et al., 2012; Pylaeva et al., 2019; Rocha et al., 2015).

53 However, type I IFNs can also inhibit neutrophil recruitment to the ganglia by suppressing

54 chemokine expression after HSV infection (Stock, Smith, \& Carbone, 2014), suggesting that the

55 interplay of IFNs and neutrophil activity may be dependent on tissue type and the pathogen. The

56 relationship between neutrophil-intrinsic type I IFN signaling and infection outcomes is less clear.

57 Type I IFNs can promote expression of interferon stimulated genes (ISGs) and pro-inflammatory 
cytokines in neutrophils, suggesting a potential role in driving immunopathology (Galani et al.,

59 2017). During Leishmania infection, however, IFNAR signaling appears to suppress neutrophil-

60 dependent killing of parasites (Xin et al., 2010), which emphasizes the complexity of IFN-

61 mediated neutrophil responses.

Genital herpes is a chronic, sexually transmitted infection that affects over 400 million

63 people worldwide (World Health Organization, 2007) and can be caused by two members of the

64 alphaherpesvirus family, herpes simplex virus-2 (HSV-2) or the related HSV-1. Genital herpes is

65 characterized by recurrent episodes of inflammation and ulceration and the factors that drive

66 disease are unclear. In humans, ulcer formation is associated with suboptimal viral control and

67 spread during episodes of reactivation (Roychoudhury et al., 2020; J. T. Schiffer \& Corey, 2013;

68 Joshua T Schiffer et al., 2013), while in mouse models, severity of disease often correlates with

69 susceptibility to infection and the level of viral replication in the genital mucosa (Gopinath et al.,

70 2018). Neutrophil infiltration into sites of active HSV-2 ulcers have also been reported in humans

71 (Boddingius, Dijkman, Hendriksen, Schift, \& Stolz, 1987), but whether these cells are helpful or

72 harmful during HSV infection is unknown. While neutrophils have been associated with tissue

73 damage after multiple routes of HSV-1 infection (Divito \& Hendricks, 2008; Khoury-Hanold et

74 al., 2016; Rao \& Suvas, 2018; Thomas, Gangappa, Kanangat, \& Rouse, 1997), a protective role

75 for neutrophils after genital HSV-2 infection has also been reported (Milligan, 1999; Milligan,

76 Bourne, \& Dudley, 2001), although use of non-specific depletion antibodies have muddled the

77 respective contribution of neutrophils and other innate immune cells such as monocytes, which are

78 known to be antiviral (Iijima, Mattei, \& Iwasaki, 2011). Furthermore, increased neutrophil

79 recruitment to the HSV-2 infected vaginal epithelial barrier resulted in greater epithelial cell death,

80 suggesting that neutrophil responses may indeed be pathogenic (Krzyzowska et al., 2014). 
81 However, the factors that distinguish pathogenic vs non-pathogenic neutrophil responses during

82 viral infection, including HSV-2 infection, remain ill-defined.

83 To address this, we evaluated the impact of neutrophils on genital disease severity using

84 two models of HSV infection that result in low levels (HSV-1) or high levels of inflammation

85 (HSV-2) (A. G. Lee et al., 2020). Between these two states, heightened expression of type I IFN

86 during the resolution phase of acute infection and sustained expression of ISGs in neutrophils was

87 detected after HSV-2 infection but not HSV-1. Therapeutic antibody-mediated blockade of

88 IFNAR1 as well as neutrophil-specific deletion of IFNAR1 reduced both genital inflammation as

89 well as vaginal IL-18 levels during the resolution phase of acute HSV-2 infection. Accordingly,

90 therapeutic neutralization of IL-18 also ameliorated genital disease after HSV-2 infection.

91 Together, our data demonstrates that sustained type I IFN signaling is a key determinant of

92 pathogenic neutrophil responses during viral infection, and identifies neutrophil- and type I IFN-

93 dependent IL-18 production as a novel driver of inflammation during genital HSV-2 infection. 


\section{RESULTS}

95 Neutrophils are a component of severe genital inflammation after vaginal HSV-2 infection.

To determine the role of neutrophils in our model of vaginal HSV-2 infection, wildtype

97 (WT) female C57BL/6 mice were treated with Depo-Provera (depot medroxyprogestrone, DMPA)

to hold mice at the diestrus phase of the estrus cycle and normalize susceptibility to infection

99 (Kaushic, Ashkar, Reid, \& Rosenthal, 2003). Neutrophils were depleted in DMPA-treated mice

101 isotype control. One day later, mice were inoculated intravaginally with 5000 plaque forming units

102 (PFU) of WT HSV-2 strain 186 syn+ (WT HSV-2). Neutrophils were effectively depleted from

103 the vagina up to 5 d.p.i. (Figure 1A). In order to focus on genital inflammation, mice were

104 monitored for 1 week after infection, as progression of disease within the second week of our

105 infection model is largely indicative of viral dissemination into the central nervous system. In both

106 cohorts, mild genital inflammation was apparent starting at 4 d.p.i. in a small fraction of mice

107 (Figure 1B). Over time, progression of disease in the neutrophil-depleted mice was significantly

108 slower compared to the controls. Remarkably, as late as 7 d.p.i., a proportion of the neutrophil-

109 depleted group remained uninflamed, in contrast to the isotype control group in which all mice

110 displayed signs of inflammation (Figure 1B). To confirm the disparity in scored disease severity,

111 we examined the vagina and genital skin by histology. At 6 d.p.i., epithelial denuding and damage

112 was apparent in the isotype control-treated mice (Figure 1C). In contrast, only a limited amount of

113 epithelial destruction was observed in neutrophil-depleted mice, with less cellular infiltrates at

114 sites of damage and in the lumen (Figure 1C). Furthermore, the epithelial layer proximal to areas

115 of damage was morphologically distinct in isotype control-treated animals compared to neutrophil-

116 depleted animals, suggesting diverse epithelial responses after infection in the presence or absence 
117 of neutrophils (Figure 1C). Similarly, destruction of the epidermis and separation of the epidermis

118 from the dermis was widespread in the genital skin of isotype control-treated mice but not in

119 neutrophil-depleted mice (Figure 1C). Unexpectedly, differences in genital inflammation and

120 mucosal damage were largely independent of changes in viral control in the absence of neutrophils,

121 as viral shedding into the vaginal lumen was similar between the two groups (Figure 1D). Indeed,

122 disease severity was decreased in neutrophil-depleted mice despite a slight delay in the resolution

123 of viral replication at 5 d.p.i. (Figure 1D).

124 We next evaluated whether the decreased inflammation after neutrophil depletion was due

125 to changes in the cellular response against HSV-2 infection. We examined the recruitment of

126 Ly6C+ monocytes, NK cells, CD4 and CD8 T cells, all of which have been implicated in either

127 the control of HSV or modulation of disease severity (A. J. Lee \& Ashkar, 2012; Shin \& Iwasaki,

128 2013; Truong, Smith, Sandgren, \& Cunningham, 2019). To remove intravascular cells and to limit

129 our analysis to cells within the vagina, tissues were thoroughly perfused prior to collection (Scott

130 et al., 2018). Unexpectedly, there was no significant difference in the number of Ly6C+CD11b+

131 cells (Figure 1 - Supplement 1A), NK cells (Figure 1 - Supplement 1B), total CD4 (Figure 1 -

132 Supplement 1C) or CD8 T cells (Figure 1 - Supplement 1D) that were recruited to the vagina over

133 the first six days after infection regardless of whether neutrophils were present or not. Thus, our

134 data demonstrate that neutrophils do not play a significant antiviral role in our model of vaginal

135 HSV-2 infection, and rather promote genital inflammation with minimal impact viral burden and

136 recruitment of other immune cells to the vagina.

138 Neutrophil extracellular trap formation and oxidative burst are not major drivers of genital

139 inflammation after $\mathrm{HSV}-2$ infection. 

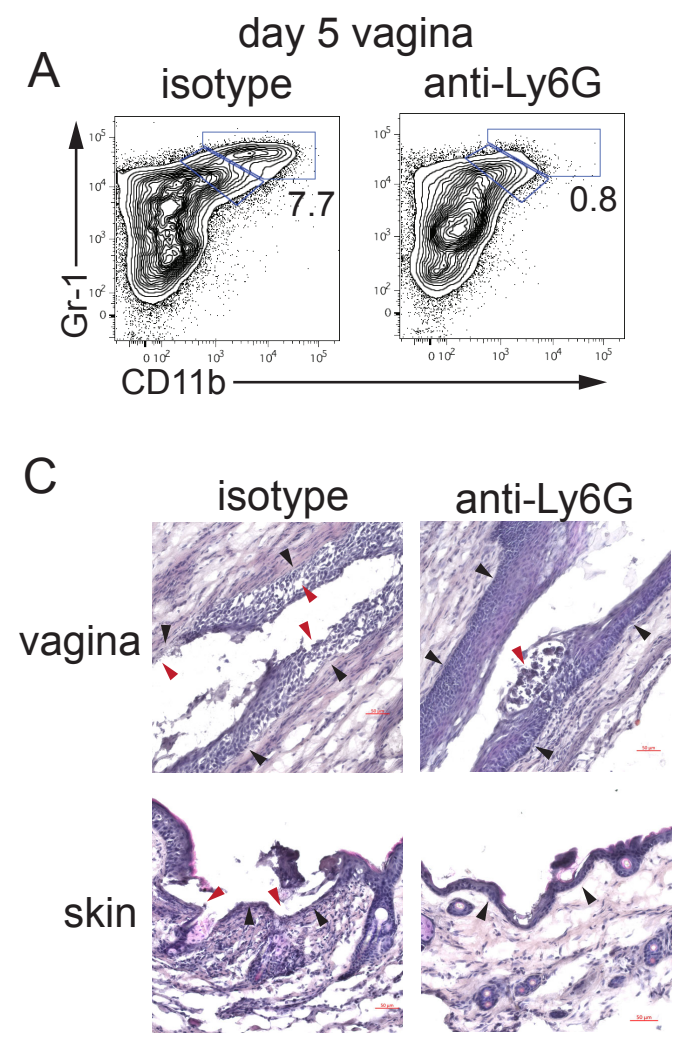
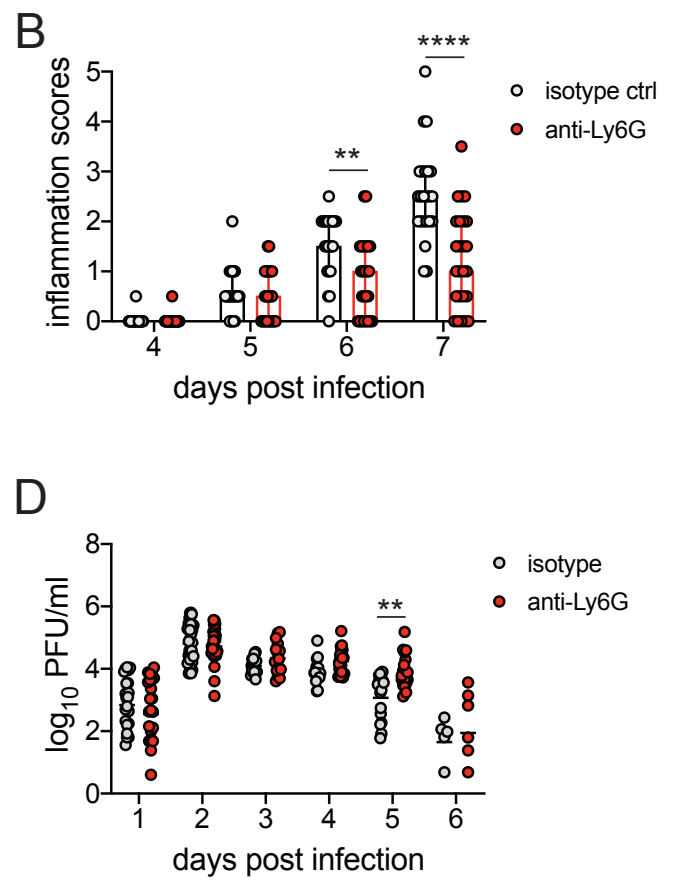

Figure 1. Neutrophil depletion reduces disease severity after HSV-2 vaginal infection. Female C57BL/6J mice were treated with DMPA and inoculated intravaginally (ivag) with 5000 PFU HSV-2. One day prior to HSV-2 inoculation, mice were injected intraperitoneally (i.p.) with 500 $\mu \mathrm{g}$ of rat IgG2a isotype control or anti-Ly6G. A. Depletion was confirmed by flow cytometry in the vagina at 5 d.p.i.. Numbers in plots show the frequency of Gr-1+CD11b+ neutrophils. B. Inflammation scores over the first 7.d.p.i. of mice treated with anti-Ly6G antibody $(n=25)$ or isotype control $(n=23)$ Mice showed no signs of disease prior to 4 d.p.i.. C. Histology of the vagina (top) or genital skin (bottom) at 6 d.p.i. from isotype control (left) or anti-Ly6G antibody-treated mice (right). Red arrows point to areas of epithelial denuding or damage, black arrows denote the basement membrane. D. Infectious virus as measured by plaque assay in vaginal washes collected daily (both groups day $1: n=22$, day $2: n=28$, day $3: n=15$, day 4: $n=16$, day 5: $n=19$, day 6: $n=6$ ). Data in $\mathbf{B}$ and $\mathbf{D}$ are pooled from 4 independent experiments. Data in $\mathbf{C}$ is representative of 2 independent experiments. Bars in $\mathbf{B}$ show median with interquartile range. Horizontal bars in $\mathbf{C}$ show mean. Statistical analysis was performed by repeated measures two-way ANOVA with Geisser-Greenhouse correction and Bonferroni's multiple comparisons test $(\mathbf{B})$ or repeated measures two-way ANOVA with Bonferroni's multiple comparison's test (D). ${ }^{* *} \mathrm{p}<0.01$, $* * * * \mathrm{p}<0.001$. Raw values for each biological replicate, epsilon values and specific $\mathrm{p}$ values are provided in Figure 1 - Source Data. 
We next wanted to determine whether neutrophil-specific effector functions were

141 promoting disease after HSV-2 infection. NETs have been associated with tissue damage in the

142 context of both infectious (C. N. Jenne \& Kubes, 2015) and non-infectious disease (Granger,

143 Peyneau, Chollet-Martin, \& de Chaisemartin, 2019). To test whether NETs play a role in genital

144 disease after HSV-2 infection, we first examined the ability of neutrophils to form NETs when

145 exposed to HSV-2. In vitro stimulation of neutrophils with HSV-2 resulted the enlargement of cell

146 nuclei and the characteristic expulsion of DNA coated in citrullinated histones, which is a key

147 characteristic of NETs (Figure 1 - Supplement 2A). The formation of NETs requires input from

148 multiple pathways, including histone citrullination by enzymes such as PAD4, which leads to

149 chromatin de-condensation and the eventual release of DNA (P. Li et al., 2010). To generate

150 animals that were specifically lacking PAD4 in neutrophils, we bred PAD4 ${ }^{\mathrm{fl} / \mathrm{fl}} \mathrm{x}$ MRP8-Cre mice

151 (PAD4 CKO). HSV-2 infection of these mice and their littermate controls demonstrated minimal

152 impact on genital inflammation (Figure 1 - Supplement 2B) or viral replication (Figure 1 -

153 Supplement 2C) in the genital mucosa. Thus, our data show PAD4 expression in neutrophils, and

154 likely NET formation, are not the mechanisms by which these cells mediate disease after HSV-2

155 infection.

156 We next tested whether ROS production by neutrophils mediated inflammation after HSV-

1572 infection. While production of ROS in neutrophils supports antimicrobial activity against a

158 variety of pathogens (Dinauer, 2019), excessive oxidative stress can be associated with tissue

159 injury (Mittal, Siddiqui, Tran, Reddy, \& Malik, 2014). We found that in vitro stimulation of

160 neutrophils with HSV-2 led to an increase in ROS production compared to unstimulated cells

161 (Figure 1 - Supplement 3A). To determine whether respiratory burst in neutrophils promoted

162 genital inflammation after HSV-2 infection in vivo, we infected mice with germline deficiency in 
163 Ncf2 (Ncf2 KO), which encodes p67 phox , a key component of the NADPH oxidase complex (Jacob

164 et al., 2017). HSV-2 infection of Ncf2 KO and heterozygous controls resulted in similar

165 progression of disease (Figure 1 - Supplement 3B) and did not alter viral titer (Figure 1 -

166 Supplement 3C). To confirm that tested neutrophil effector functions, including ROS production,

167 we infected mice in which the calcium-sensing molecules STIM1 and STIM2 were deleted from

168 neutrophils (Clemens, Chong, Grimes, Hu, \& Lowell, 2017). Stim1 ${ }^{\mathrm{fl} / \mathrm{fl}}$ x Stim2 ${ }^{\mathrm{fl} / \mathrm{fl}}$ x MRP8-Cre

169 (STIM1/2 DKO) mice were infected with HSV-2 and monitored for disease. As expected, there

170 was little difference in genital inflammation severity between the STIM1/2 DKO and Cre- controls

171 (Figure 1-Supplement 3D) or viral titers (Figure 1 - Supplement 3E). Together, our data show that

172 ROS production from neutrophils and other cell types play little role in driving genital

173 inflammation after HSV-2 infection.

174

175 A type I IFN signature distinguishes neutrophil responses after genital HSV-1 and HSV-2

176 infection.

177 To identify the factors that drove pathogenic neutrophil responses after HSV-2 infection,

178 we turned to a complementary model of HSV-1 genital infection that we had previously described

179 (A. G. Lee et al., 2020). Inoculation with the same dose of HSV-1 and HSV-2 led to profound

180 differences in genital inflammation (Figure 2A) despite comparable levels of mucosal viral

181 shedding (A. G. Lee et al., 2020). Importantly, magnitude of the neutrophil response in the vagina

182 was similar between HSV-1 and HSV-2 infected mice during the course of acute mucosal infection

183 (Figure 2B), and neutrophils could be found infiltrating sites of both HSV-1 and HSV-2-infected

184 epithelium, although it appeared that the interaction between neutrophil and virally infected

185 epithelial cells after HSV-1 inoculation was not as extensive as after HSV-2 inoculation (Figure 

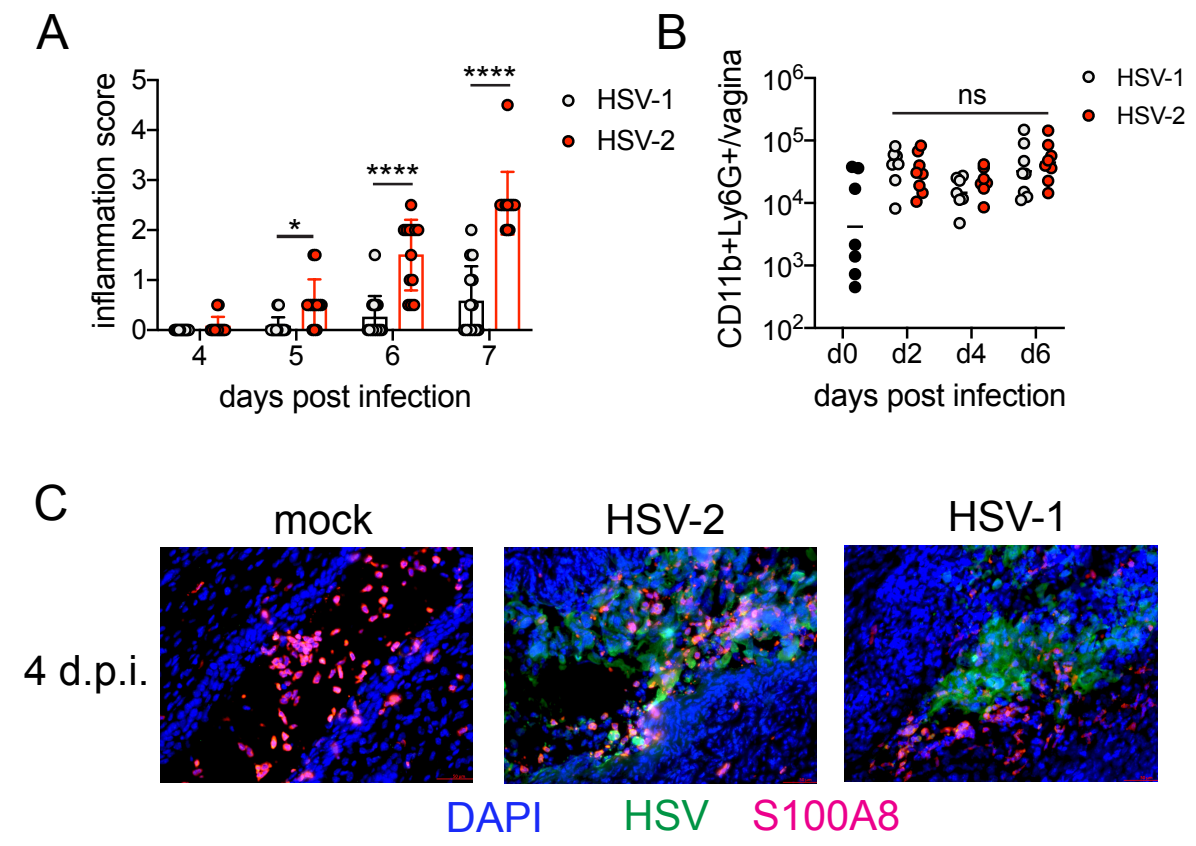

Figure 2. Magnitude of the neutrophil response during HSV-1 and HSV-2 is similar despite difference in disease outcome. Female C57BL/6J mice were treated with DMPA and inoculated ivag with $10^{4}$ PFU HSV-1 McKrae or HSV-2. A. Inflammation scores were monitored for 7 d.p.i.. For HSV-1: $\mathrm{n}=14, \mathrm{HSV}-2: \mathrm{n}=13$. B. Neutrophils were counted by flow cytometry in vaginal tissues at the indicated days after HSV-1 or HSV-2 infection. For $\mathrm{d} 0: \mathrm{n}=7$, day $2: \mathrm{n}=8$, day $4: \mathrm{n}=7$, day $6: \mathrm{n}=8$. C. Vaginas were harvested from PBS inoculated (mock), HSV-1 or HSV-2 inoculated mice at 4 d.p.i., and tissue sections were probed with antibodies against HSV proteins (green) or S100A8 (red). DAPI (blue) was used detect cell nuclei. Images are representative of six mice per group. Data are pooled from 3 (A) or 2 (B, C) independent experiments. Bars show median with interquartile range in $\mathbf{A}$ and mean in $\mathbf{B}$. Statistical significance was measured by repeated measures two-way ANOVA with Geisser-Greenhouse correction and Bonferroni's multiple comparisons test $(\mathbf{A})$ or two-way ANOVA with Bonferroni's multiple comparisons test $(\mathbf{B}) .{ }^{*} \mathrm{p}<0.05, * * * * \mathrm{p}<0.001, \mathrm{~ns}=$ not significant. Raw values for each biological replicate, epsilon values and specific $\mathrm{p}$ values are provided in Figure 2 - Source Data. 
2C). In contrast to HSV-2 infection, antibody-mediated depletion of neutrophils with anti-Ly6G antibody prior to inoculation with HSV-1 did not reduce the development of genital inflammation during the first 7 days after infection (Figure 2 - Supplement 1). Together, our data suggests that the regulation of the neutrophil response after HSV-1 or HSV-2 infection was distinct, leading to disparate inflammatory outcomes.

To better better understand the differences between pathogenic neutrophil responses after

192 HSV-2 infection and the non-pathogenic neutrophil responses after HSV-1, we performed single

193 cell RNA-sequencing (scRNA-seq) on sorted live vaginal cells from a mock infected mouse or

194 mice infected with HSV-1 or HSV-2 using the 10x Genomics platform (Zheng et al., 2017). Each

195 sample was comprised of cells from a single animal in to better delineate potential subsets within

196 cell populations, particularly neutrophils. Analysis across 21,633 cells in all samples revealed 17

197 unique clusters in the vagina during HSV infection after filtering, including myeloid cells,

198 lymphocytes and epithelial cells (Figure 3A). Neutrophils were identified by expression of known

199 cell markers such as S100a8 and Csf3r (Figure 3B). In mock infected animals, the vaginal

200 neutrophil population was dominated by cluster 0 , and upon infection, at least two additional

201 neutrophil subsets, cluster 2 and cluster 5, were clearly present (Figure 3C). While HSV-1 infected

202 mice retained all three subpopulations of neutrophils in the vagina at 5 d.p.i., in HSV-2 infected

203 mice the presence of cluster 0 was greatly reduced and the bulk of the neutrophils was composed

204 of cluster 2 and 5 (Figure 3C). One major distinguishing characteristic between "homeostatic"

205 cluster 0 and "infection" clusters 2 and 5 was the extent of ISG expression, in which cluster 0

206 expressed low levels of genes associated with a type I IFN response, even in infected animals,

207 while clusters 2 and 5 expressed high levels of these genes (Figure 3D) (Liberzon et al., 2015).

208 Furthermore, expression of ISGs within clusters 2 and 5 was higher after HSV-2 infection 
A

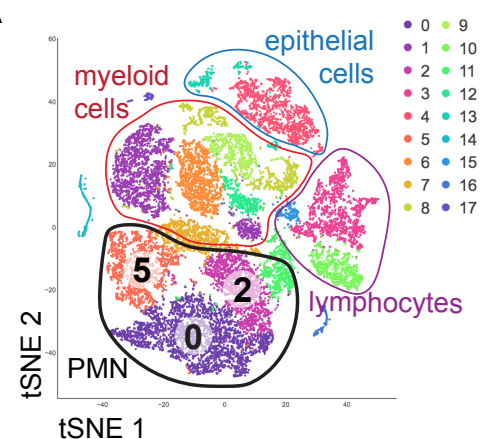

B

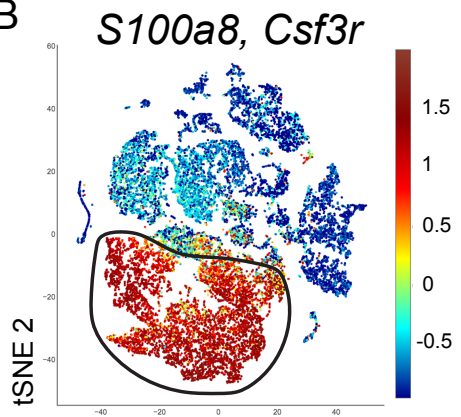

tSNE 1

C

mock

\section{HSV-1}

HSV-2

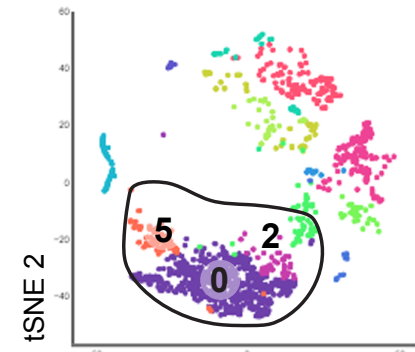

tSNE 1

IFN response

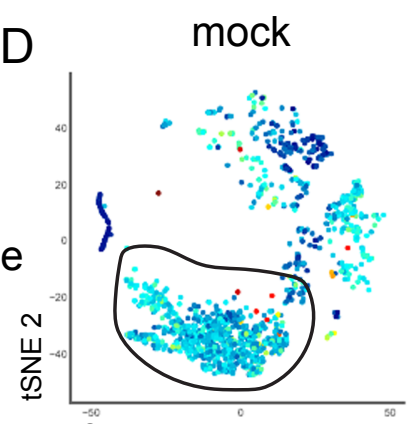

tSNE 1
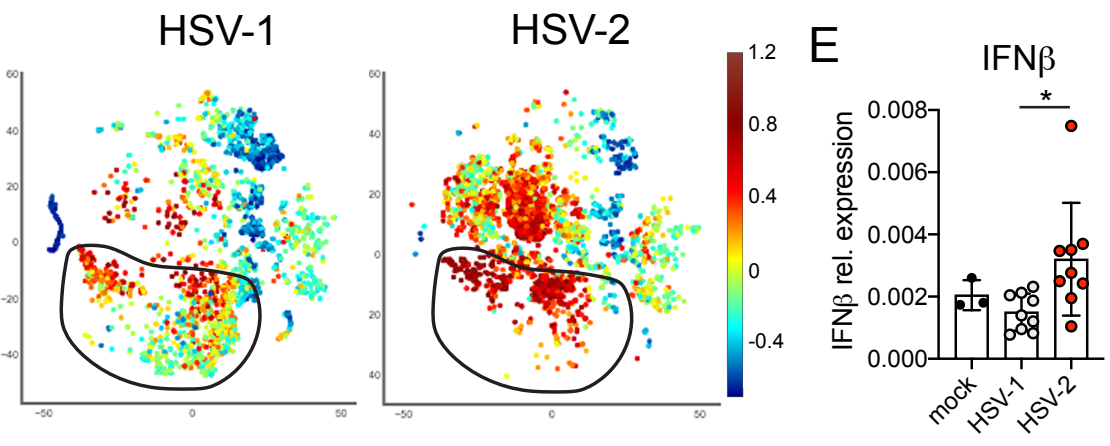

Figure 3. Single cell transcriptome analysis reveals a sustained IFN signature in the neutrophil response against HSV-2. Mice were infected as described in Figure 2. Vaginas were harvested at 5 d.p.i., live cells were flow sorted and subjected to high-throughput scRNA-seq. A. A t-Distributed Stochastic Neighbor Embedding (tSNE) visualization of 21,633 cells across all mice resolves 17 distinct clusters in the vaginal tissue. Clusters can be identified as myeloid cells (red border), epithelial cells (blue border) or lymphocytes (purple border). Neutrophils are encircled in black, and contain three distinct clusters ( 0,2 and 5). B. Neutrophils are defined by high expression of S100A8 and G-CSFR (Csf3r). C. tSNE plots of vaginal cell clusters from mock inoculated or HSV-infected mice. Neutrophil populations are circled in black. D. Distribution of expression for genes within the Hallmark IFN $\alpha$ Response gene set. E. Expression of IFN $\beta$ transcripts normalized to RPL13 in mock inoculated $(n=3)$ mice or mice at 5 d.p.i. with HSV-1 (n=9) or HSV-2 ( $\mathrm{n}=9$ ) as measured by qRT-PCR. scRNA-seq in A-D was performed once. Data in $\mathbf{E}$ are pooled from 2 independent experiments. Statistical significance was measured by one-way ANOVA with Tukey's multiple comparisons test. $* p<0.05$. Raw values for each biological replicate and specific $p$ values for $E$ are provided in Figure 3 - Source Data. 
compared to HSV-1 (Figure 3C), which was confirmed by quantitative RT-PCR (qRT-PCR) analysis of select ISGs that are differentially expressed in the vagina at 5 days after HSV-1 or

211 HSV-2 infection (Figure 3 - Supplement 1). qRT-PCR shows that expression of CXCL10 (Figure

2123 - Supplement 1A, B) and Gbp2 (Figure 3 - Supplement 1C, D) is increased in HSV-2 infected

213 vaginas compared to HSV-1, while IL-15 is not (Figure 3 - Supplement 1E, F), which supports the

214 accompanying scRNA-seq analysis. While type I IFN was robustly produced early during acute

215 infection after both HSV-1 and HSV-2 infection (Figure 3 - Supplement 2), greater expression of

216 IFN $\beta$ was detected in the vagina after HSV-2 infection compared to HSV-1 at time points

217 corresponding to the onset of genital inflammation (Figure 3E). Thus, during viral infection,

218 distinct neutrophil subsets can be classified by transcriptional profiling, and expression of ISGs

219 suggest that a key difference between a pathogenic and non-pathogenic neutrophil response during 220 viral infection may be sustained IFN signaling. during $H S V-2$ infection.

We next wanted to test whether type I IFN signaling promoted immunopathology during genital HSV-2 infection. IFNAR1-deficient mice are highly susceptible to HSV regardless of the route of inoculation (Gill, Deacon, Lichty, Mossman, \& Ashkar, 2006; Iversen, Ank, Melchjorsen,

227 \& Paludan, 2010; Iversen et al., 2016; Reinert et al., 2012; Royer et al., 2019; Svensson, Bellner, 228 Magnusson, \& Eriksson, 2007; Wilcox, Folmsbee, Muller, \& Longnecker, 2016), and rapidly 229 succumb to infection, mainly due to a loss of viral control. To investigate the temporal effects of 230 type I IFNs in HSV-2 genital disease, we used an antibody against IFNAR1 to block the receptor 231 at different time points after infection (Scott et al., 2018). When mice were injected i.p. with anti- 
232 IFNAR1 antibody on the day of HSV-2 inoculation, disease progression was more rapid compared

233 to isotype control treated animals (Figure 4 - Supplement 1A), and mice succumbed to infection

234 at a faster rate (Figure 4 - Supplement 1B), in a manner similar to IFNAR1-deficient mice (Iversen

235 et al., 2010; Iversen et al., 2016; A. J. Lee et al., 2017; Reinert et al., 2012; Wang et al., 2012).

236 Inflammation and rapid disease progression were likely due to significantly elevated viral burden

237 in the anti-IFNAR1 antibody treated mice compared to isotype controls (Figure 4 - Supplement

238 1C), as HSV is a highly lytic virus that is capable of independently inducing epithelial tissue

239 damage (Horbul, Schmechel, Miller, Rice, \& Southern, 2011). To focus on the effects of persistent

240 IFN signaling in the vagina after HSV-2 infection, we also treated mice with a single injection of

241 anti-IFNAR1 antibody or an isotype control at 4 d.p.i.. In stark contrast to early anti-IFNAR1

242 antibody treatment, one treatment with therapeutic IFNAR1 blockade led to a significant reduction

243 in the severity of inflammation compared to isotype controls (Figure 4A). Histology of vaginal

244 tissues from isotype-treated controls at 6 d.p.i. showed widespread epithelial denuding and

245 immune cell infiltrates within the epithelial layer of the vagina (Figure 4B). In contrast, damage to

246 the vaginal epithelium in anti-IFNAR1 antibody-treated mice appeared to be localized (Figure 4B),

247 similar to neutrophil-depleted mice (Figure 1C). Similarly, the genital skin of isotype control-

248 treated mice displayed signs of severe inflammation and destruction of the epidermis, while the

249 skin structure of anti-IFNAR1 antibody-treated mice was largely intact (Figure 4B). Furthermore,

250 IFNAR1 blockade at 4 d.p.i. had little impact on mucosal viral shedding (Figure 4C). Collectively,

251 these data show that the protective effect of type I IFN on control of genital HSV infection is

252 limited to the early stages of acute infection, and that sustained IFN signaling in the later stages of

253 acute HSV-2 genital infection drive inflammation with minimal effect on viral replication. 
A
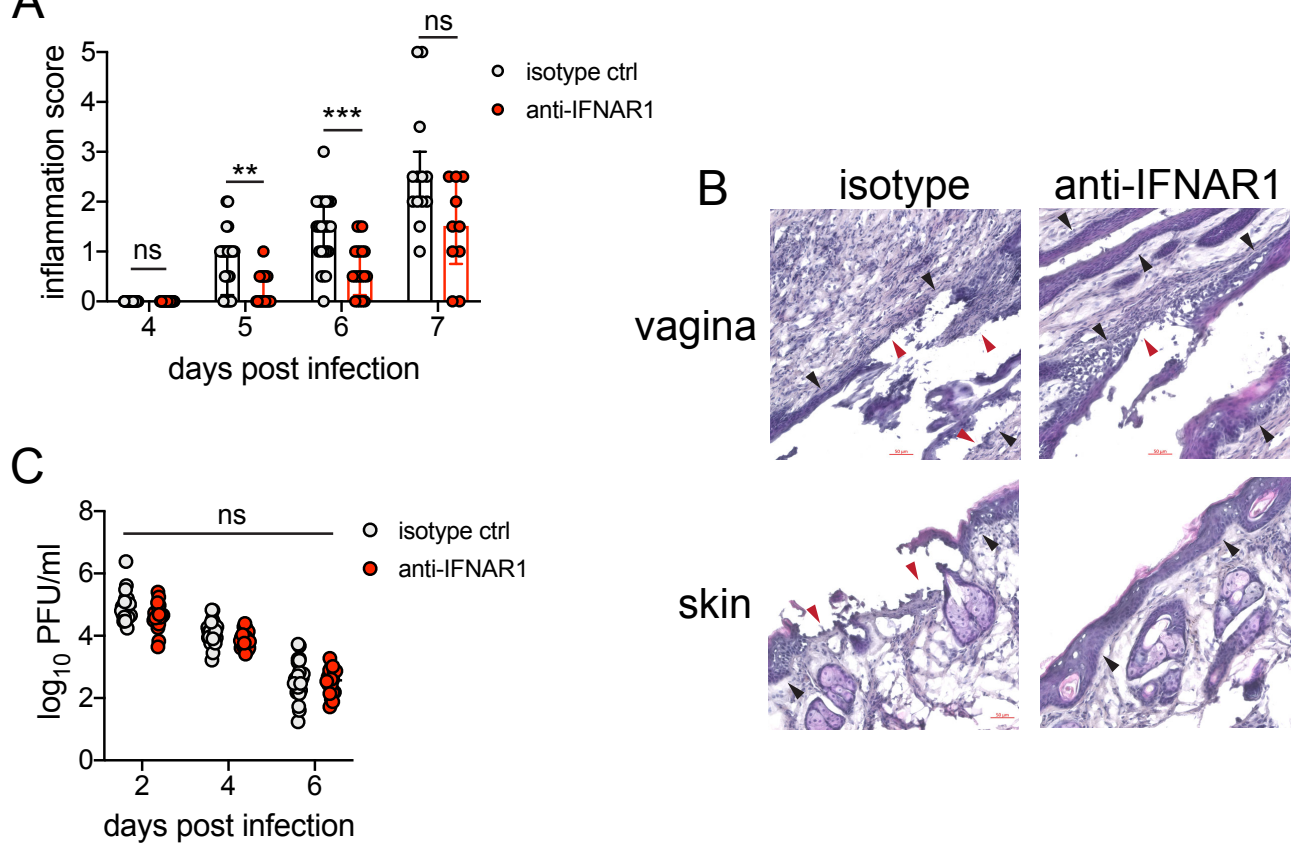

Figure 4. Inhibition of type I IFN signaling during the resolution phase of infection reduces inflammation after HSV-2 infection. Mice were infected as described in Figure 2. At 4 d.p.i., mice were injected i.p. with either $1 \mathrm{mg}$ of anti-IFNAR1 antibody $(n=10-13)$ or isotype control $(n=7-9)$ and monitored for disease progression. Mice showing overt signs of genital inflammation at the time of antibody injection (4 d.p.i.) were excluded from the study. A. Inflammation scores of antibody-treated mice over the first 7 d.p.i.. B. Histology of the vagina (top) or genital skin (bottom) at 6 d.p.i. Red arrows point to areas of epithelial denuding or damage. Black areas denote the basement membrane. $\mathbf{C}$. Infectious virus as measured by plaque assay in vaginal washes collected on the indicated days. Data are pooled from (A, C) or representative of 3 independent experiments. Bars in A show median with interquartile range, bars in $\mathbf{C}$ show mean. Statistical significance was measured by repeated measures two-way ANOVA with Geisser-Greenhouse correction and Bonferroni's multiple comparisons test (A) or two-way ANOVA with Bonferroni's multiple comparisons test $(\mathbf{C}) .{ }^{*} \mathrm{p}<0.05,{ }^{*} \mathrm{p}<0.01,{ }^{*} * \mathrm{p}<0.005$, $\mathrm{ns}=$ not significant. Raw values for each biological replicate, epsilon values and specific $\mathrm{p}$ values are provided in Figure 4 - Source Data. 
Single-cell transcriptional profiling data suggested that type I IFN signaling was highly

255 robust in vaginal neutrophils after HSV-2 infection (Figure 3D). To determine whether intrinsic

256 IFN signaling in neutrophils promoted immunopathology, we deleted the type I IFN receptor from

257 granulocytes by breeding IFNAR1 ${ }^{\mathrm{fl} / \mathrm{fl}} \mathrm{x}$ MRP8-Cre mice (IFNAR1 CKO). After confirming that

258 IFNAR1 ablation was limited to the neutrophil population (Figure 5A), IFNAR1 CKO mice and

259 littermate Cre- controls were vaginally infected with HSV-2. Despite differences in IFNAR1

260 expression, the number of neutrophils recovered from the vaginal lumen was similar between the

261 IFNAR1 CKO mice and their Cre- control littermates (Figure 5B). Strikingly, although the

262 magnitude of the vaginal neutrophil response was similar, we found that the severity of genital

263 inflammation presented by the IFNAR1 CKO mice was significantly reduced compared to the Cre-

264 controls (Figure 5C). As observed after neutrophil depletion, a subset of the IFNAR1 CKO cohort

265 did not develop any signs of inflammation as late as 7 d.p.i. (Figure 5C). Similar to our

266 observations with therapeutic IFNAR1 blockade, IFNAR1 CKO mice exhibited less pathology in

267 both the vagina and genital skin compared to Cre- controls. (Figure 5D). Distinct disease outcomes

268 between the Cre- controls and IFNAR1 CKO mice occurred independently of viral control, as viral

269 load in the mucosa were similar between the two groups (Figure 5E). Together, our data

270 demonstrates that tissue inflammation during HSV-2 infection is largely driven by prolonged type

271 I IFN production, which acts directly upon neutrophils to drive disease.

272

273 Sustained type I IFN signaling and neutrophils regulate production of pathogenic IL-18 in the

274 vagina during $\mathrm{HSV}-2$ infection.

275 Type I IFN stimulation of neutrophils can upregulate ISGs as well as several pro276 inflammatory cytokines (Galani et al., 2017). To determine whether type I IFN was driving disease 

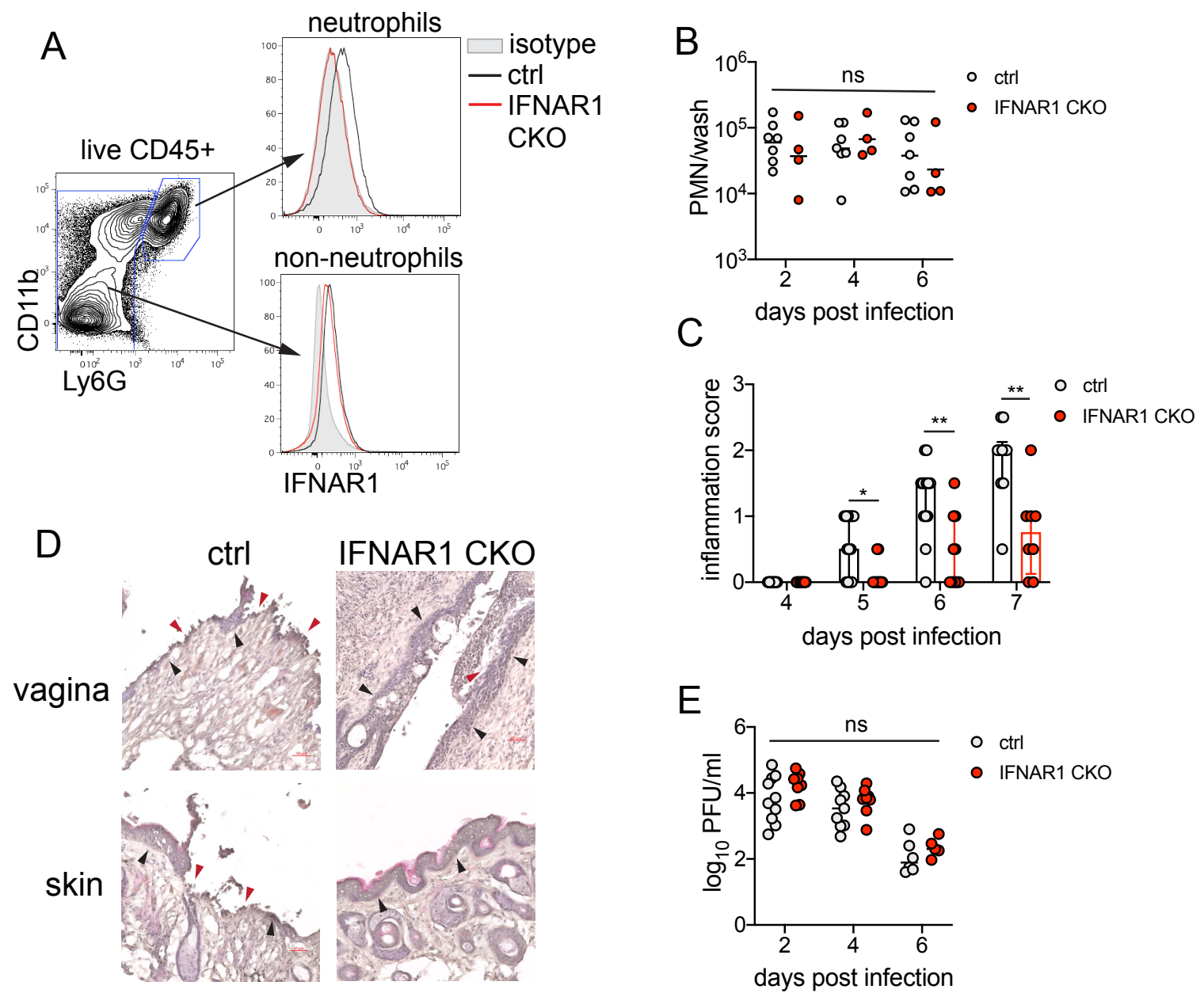

Figure 5. Type I IFN signaling in neutrophils promotes genital inflammation after HSV-2 infection. A. IFNAR1 expression on neutrophils and non-neutrophil hematopoietic cells from the bone marrow of naive IFNAR ${ }^{\mathrm{t} / \mathrm{fl}} \mathrm{x}$ MRP8-Cre (IFNAR1 CKO) or Cre- littermate controls. Plot is gated on live CD45+ cells. CD11b+Ly6G+ cells are neutrophils, Ly6G- cells are non-neutrophils. Gray histogram shows isotype staining, black open histogram is Cre- control, and red open histogram is IFNAR1 CKO. B. Neutrophils were counted by flow cytometry in vaginal washes collected at the indicated days from IFNAR1 CKO $(n=4)$ or Cre- controls $(n=7)$ that were infected with HSV-2 as described in Figure 1. C. Inflammation scores for the first 7 d.p.i. of IFNAR1 CKO $(n=10-13)$ or Cre- controls $(n=8-11)$. D. Histology on vagina and genital skin at 6 d.p.i.. Red arrows point to areas of epithelial denuding or damage, black arrows denote basement membrane E. Infectious virus as measured by plaque assay from vaginal washes collected on the indicated days from IFNAR1 CKO $(n=5-8)$ or Cre- controls $(n=7-10)$. Data in $\mathbf{C}$ and $\mathbf{E}$ are pooled from 3 independent experiments, data in $\mathbf{B}$ are pooled from 2 independent experiments, and data in $\mathbf{D}$ are representative of 2 independent experiments. Bars in $\mathbf{C}$ show median with interquartile range, bars in $\mathbf{B}$ and $\mathbf{E}$ show mean. Statistical significance was measured by mixed-effects analysis with $(\mathbf{C})$ or without $(\mathbf{B}, \mathbf{E})$ Geisser-Greenhouse correction and Bonferroni's multiple comparisons test. $* \mathrm{p}<0.05,{ }^{*} \mathrm{p}<0.01$, ns $=$ not significant. Raw values for each biological replicate, epsilon values and specific $\mathrm{p}$ values are provided in Figure 2 - Source Data. 
277 by shaping the cytokine milieu within the vagina, we first measured several pro-inflammatory

278 cytokines in the vagina at 5 d.p.i., in the presence or absence of neutrophils. The production of

279 inflammatory cytokines such as IL-6 (Figure 6 - Supplement 1A), IL-1 $\beta$ (Figure 6 - Supplement

280 1B) or TNF (Figure 6 - Supplement 1C), all of which have been associated with genital

281 inflammation and HSV-2 infection in humans (Gosmann et al., 2017; Masson et al., 2014; Murphy

282 \& Mitchell, 2016), was similar between both neutrophil-depleted and control groups. Production

283 of IFN $\gamma$ (Figure 6 - Supplement 1D) as well as IL-12p70 (Figure 6 - Supplement 1E), both

284 cytokines associated with a type I immune response and important for HSV control, were similar

285 between the neutrophil-depleted and control groups. However, when we measured IL-18, an IL-1

286 family cytokine that primarily known for mediating innate defense (Harandi, Svennerholm,

287 Holmgren, \& Eriksson, 2001) and for promoting IFN $\gamma$ production from NK cells during genital

288 HSV-2 infection (A. J. Lee et al., 2017), we detected a notable difference between neutrophil-

289 depleted and control mice (Figure 6A), suggesting an unexpected role for this cytokine in driving

290 disease during HSV-2 infection.

291 To determine whether type I IFN signaling regulated IL-18 production in the vagina, we

292 assessed IL-18 levels in the vaginal lumen after therapeutic antibody-mediated IFNAR1 blockade.

293 At 5 d.p.i., similarly to neutrophil-depleted mice, we found that IL-18 levels were markedly

294 reduced (Figure 6B). Importantly, measurement of IL-18 in the vagina of IFNAR1 CKO at 5 d.p.i.

295 also revealed a significant decrease in cytokine levels compared to littermate controls (Figure 6C).

296 To determine whether IL-18 was playing a key role in driving immunopathology during genital

297 HSV-2 infection, we therapeutically administered an IL-18 neutralizing antibody to HSV-2

298 infected animals starting at 3 d.p.i.. Remarkably, neutralization of IL-18 led to a considerable

299 reduction in disease severity (Figure 6D), without any impact on viral control (Figure 6E). To 
300 determine the source of pathogenic IL-18 in the vagina, we probed vaginal tissues for the

301 neutrophil marker S100A8 and IL-18 at 6 d.p.i. (Figure 6F). Detection of IL-18 and S100A8

302 around a single nucleus demonstrated that neutrophils could be a source of IL-18 during vaginal

303 HSV-2 infection (Figure 6F). However, we also identified IL-18-reactive cells that were negative

304 for S100A8 but in close proximity to neutrophils (Figure 6F), suggesting the potential for multiple

305 cellular sources of IL-18. Thus, our data demonstrate that sustained type I IFN signaling in

306 neutrophils leads to the production of vaginal IL-18, and reveal IL-18 to be a novel regulator of

307 disease after HSV-2 infection. 
bioRxiv preprint doi: https://doi.org/10.1101/2020.12.20.423690; this version posted December 23, 2020. The copyright holder for this preprint (which was not certified bApeer review) is the author/fund $\$$ who has granted bioRxiv a lidense to display, the preprint in perpetuity. It is made

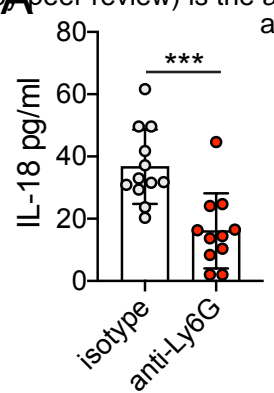

D

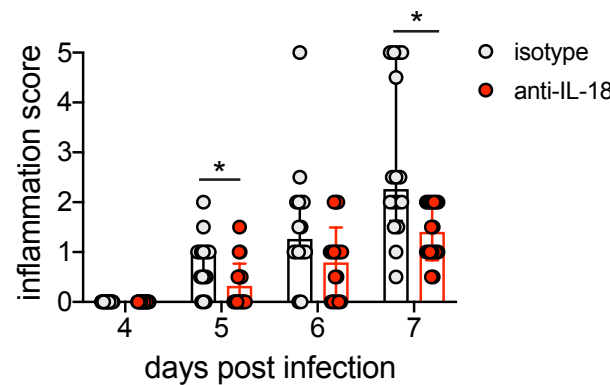

E
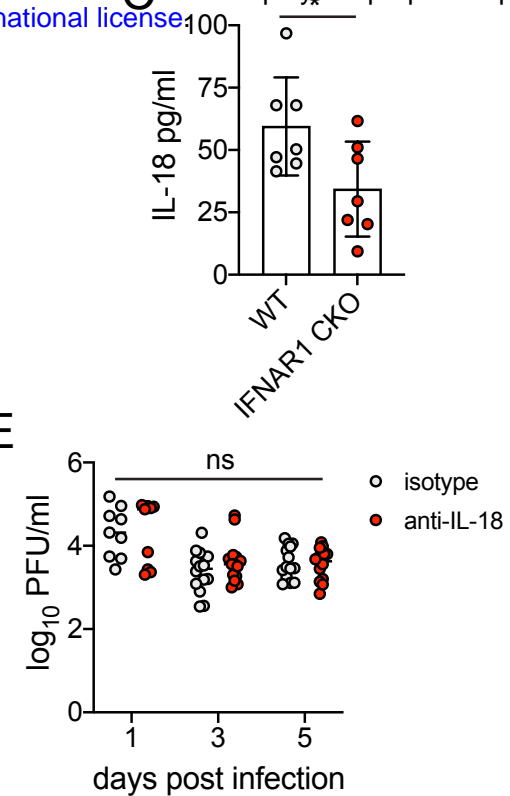
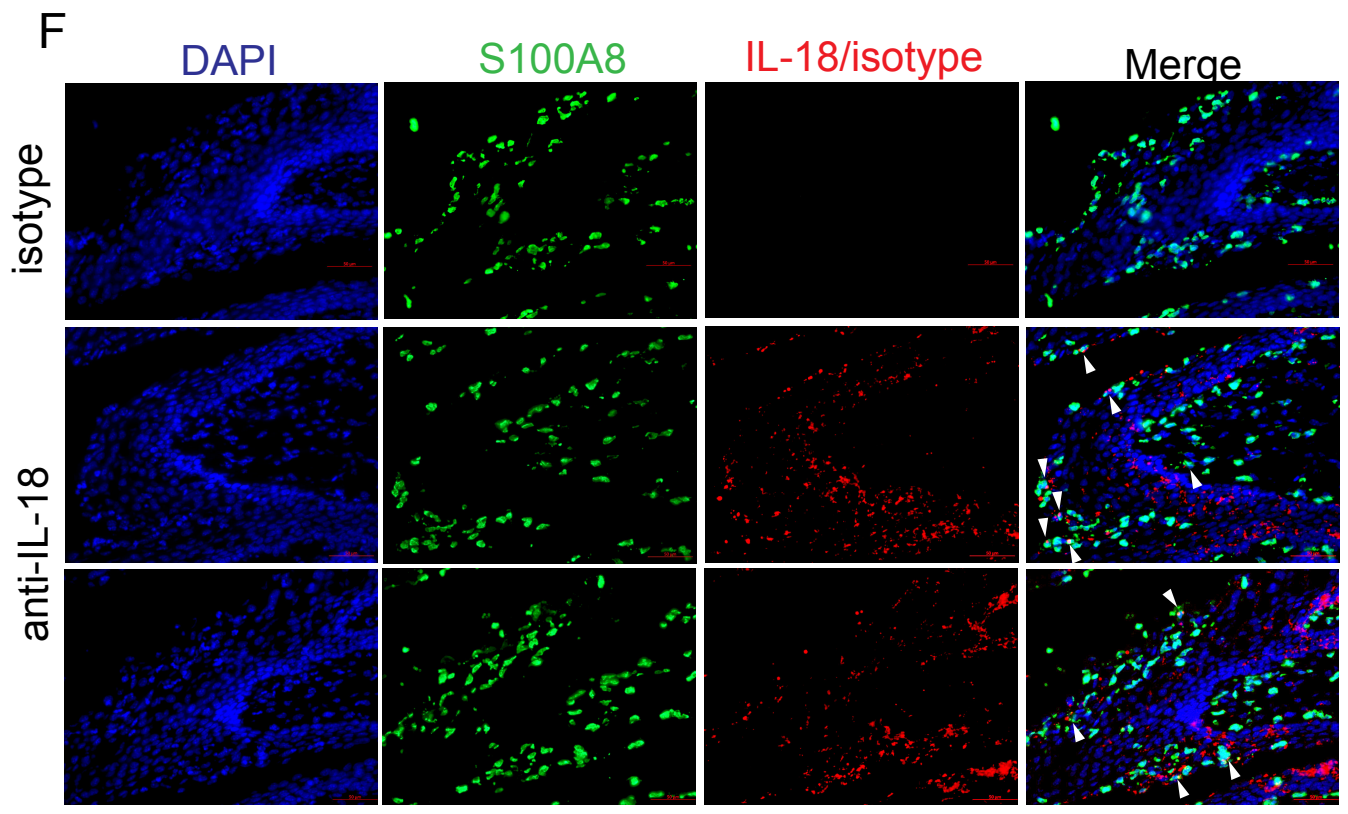

Figure 6. Sustained type I IFN signaling and neutrophils regulate pathogenic IL-18 levels in the vagina. $\mathrm{C} 57 \mathrm{BL} / 6$ mice were treated with anti-Ly6G $(\mathrm{n}=11)$ or isotype control $(\mathrm{n}=12)$ as described in Figure $1(\mathbf{A})$, therapeutically treated with anti-IFNAR1 $(n=12)$ or isotype control $(n=14)$ as described in Figure 5 (B) and infected with HSV-2, or IFNAR1 CKO $(n=7)$ and Cre- controls $(n=7)$ were infected with HSV-2 as described in Figure 5 (C). A-C. Vaginal IL-18 levels were measured by ELISA in washes collected at 5 d.p.i.. 100 $\mu$ g of anti-IL-18 neutralizing antibody $(n=18)$ or isotype control $(n=16)$ was administered ivag at 3, 4 and 5 d.p.i.. D. Inflammation scores of antibody-treated mice over the first 7 d.p.i.. E. Infectious virus as measured by plaque assay in vaginal washes collected on the indicated days $(\mathrm{n}=9-14)$. F. Immunofluorescent staining of vaginal tissues collected at 6 d.p.i.. Green shows S100A8 (neutrophil), red shows IL-18 or isotype, blue is DAPI. Top row shows tissues probed with isotype control, and bottom two rows show two representative images of tissues probed with anti-IL-18 antibody. White arrows point to IL-18+ neutrophils. Data in A-C, $\mathbf{D}$ and $\mathbf{E}$ are pooled from 4 independent experiments for each experimental setup. Data in $\mathbf{F}$ is representative of 2 independent experiments. Bars in A-C show mean and SD, bars in $\mathbf{D}$ show median with interquartile range, and bars in E show mean. Statistical significance was measured by unpaired t-test (A-C), repeated measures two-way ANOVA with (D) Geisser-Greenhouse correction and Bonferroni's multiple comparisons test, or mixed-effects analysis with Bonferroni's multiple comparisons test (D). ${ }^{*} \mathrm{p}<0.05$, ${ }^{*} * \mathrm{p}<0.005$, ns $=$ not significant. Raw values for each biological replicate, epsilon values and specific $p$ values are provided in Figure 2 - Source Data. 


\section{DISCUSSION}

In this study, we evaluated drivers of a pathogenic neutrophil response using a mouse model for an important human infection. We found that neutrophils promote genital inflammation and do not impact antiviral activity after genital HSV-2 infection, suggesting that the neutrophil response is primarily immunopathogenic. Depletion of neutrophils led to a significant decrease in

313 disease severity without affecting recruitment of other immune cells or the production of common

314 pro-inflammatory cytokines, and deficiency in genes controlling neutrophil effector functions such as ROS production and NET formation had little impact on progression of disease. Comparative analysis of single-cell transcriptional profiles revealed a strong type I IFN signature that was sustained in neutrophils responding to a highly inflammatory genital HSV-2 infection but not a

318 less inflammatory HSV-1 infection. In contrast to antibody-mediated blockade of IFNAR1 at the

319 time of infection, which led to significantly worse disease outcomes, IFNAR1 blockade just prior

320 to the resolution phase of acute mucosal infection significantly delayed the progression of genital

321 inflammation. Importantly, neutrophil-specific deficiency of IFNAR1 markedly reduced the

322 severity of genital disease after HSV-2 infection, suggesting that persistent IFN signaling drove

323 disease primary by acting on neutrophils. Ultimately, this sustained type I IFN signaling in

324 neutrophils promoted the production of pro-inflammatory IL-18, and therapeutic neutralization of

325 this cytokine also ameliorated disease. Together, our results suggest an axis of type I IFNs, neutrophils, and IL-18 as key drivers of genital disease in a mouse model of HSV-2 infection, and

327 that sustained type I IFN signaling is a key factor in distinguishing between pathogenic and non328 pathogenic neutrophil responses during mucosal viral infection. 
331 al., 2013), human immunodeficiency virus (HIV) (Meier et al., 2009; Rotger et al., 2010; Sedaghat

332 et al., 2008; Taleb et al., 2017) and simian immunodeficiency virus (SIV) (Harris et al., 2010;

333 Jacquelin et al., 2009), reveal the detrimental effect of overexuberant or sustained type I IFN

334 signaling. Notably, prolonged IFN signaling during chronic viral infection can promote

335 immunosuppression through multiple cellular and molecular mechanisms, and deletion or

336 blockade of IFNAR1 during chronic LCMV infection can alleviate immunosuppression and

337 enhance long-term viral control (Cheng et al., 2017; Taleb et al., 2017; Teijaro et al., 2013; Wilson

338 et al., 2013). However, unlike the LCMV model, early blockade of type I IFN signaling led to

339 more severe disease and a complete loss of viral control after HSV-2 infection, similar to infections

340 performed on an IFNAR1-deficient genetic background (Iversen et al., 2010; Iversen et al., 2015;

341 A. J. Lee et al., 2017; Leib et al., 1999; Reinert et al., 2012; Wang et al., 2012) and indicating an

342 early antiviral role (A. J. Lee et al., 2017; Luker, Prior, Song, Pica, \& Leib, 2003). Rather, only

343 inhibition of sustained IFN signaling led to diminished disease with minimal impact on viral

344 control, thus revealing distinct antiviral and immunopathological effects of type I IFN signaling

345 that are temporally regulated and had heretofore been unappreciated during HSV-2 infection. The

346 source of sustained type I IFN production that promotes immunopathology after genital HSV-2

347 infection is currently unknown. HSV encodes numerous proteins that can suppress type I IFN

348 production and regulate the signaling pathways (Christensen et al., 2016; Lin \& Zheng, 2019;

349 Melroe, DeLuca, \& Knipe, 2004), suggesting that production of type I IFN likely occurs from a

350 cell type that is not directly infected. While plasmacytoid dendritic cells (pDC) are known as robust

351 producers of type I IFN, previous studies have demonstrated a limited role for pDCs in genital

352 HSV-2 infection (Swiecki, Wang, Gilfillan, \& Colonna, 2013), indicating that there may be an

353 alternative source of type I IFN, such as conventional DCs (Wilson et al., 2013). 
354 In humans, type I IFN can be detected at active lesion sites during recurrent episodes (Peng et al.,

355 2009; Roychoudhury et al., 2020), although levels do not correlate with restriction of viral

356 replication (Roychoudhury et al., 2020). This raises the possibility that type I IFN induction may

357 not be antiviral and could contribute to ulcer formation, although this hypothesis has yet to be

358 tested. Human neutrophils from females are also reported to be hyper-responsive to type I IFNs

359 (Gupta et al., 2020). Although clinical disease recurrence rates between men and women with

360 genital herpes are similar (Wald et al., 2002), differences in neutrophil sensitivity to type I IFN

361 may have implications for sex-dependent mechanisms of ulcer development.

362 Type I IFN signaling orchestrates a network of ISGs that limit viral infection using a wide

363 variety of mechanisms. In vitro stimulation of neutrophils with type I IFN leads to the upregulation

364 of many common ISGs as well as inflammatory genes, including IL-18 (Galani et al., 2017).

365 Importantly, it has been suggested that type I IFN can differentially regulate expression IL-18 and

366 IL-1 $\beta$, another IL-1 family cytokine that depends on caspase-mediated cleavage for activation

367 (Zhu \& Kanneganti, 2017). It is currently unclear whether neutrophils are directly producing this

368 cytokine in our model of infection and whether IL-18 production is dependent on the classical

369 inflammasome-dependent route. As HSV also encodes proteins that can inhibit inflammasome

370 activity, including VP22 (Maruzuru et al., 2018), one possibility is that that the direct source of

371 IL-18 is a cell type that is not productively infected with HSV, such as neutrophils. Alternatively,

372 neutrophil proteases released in the extracellular space have been reported to cleave and activate

373 proIL-1 cytokines that are secreted by other cells in a caspase-1-independent manner (Clancy et

374 al., 2018; Robertson et al., 2006; Sugawara et al., 2001), suggesting a mechanism by which

375 neutrophils may modulate IL-18 levels without directly producing the cytokine themselves. Our

376 data show that along with neutrophils, IL-18 could be detected in the epithelium in cells that are 
377 in close proximity to infiltrating neutrophils, indicating that there may be multiple sources and

378 mechanisms by which pathogenic IL-18 is produced during HSV-2 infection. Understanding these

379 mechanisms could further reveal novel, specific targets for therapeutics aimed at reducing

380 inflammation during genital herpes, and as such, are currently under investigation.

381 During HSV-2 vaginal infection, IL-18 stimulates NK cells to mediate rapid antiviral IFN $\gamma$

382 production upon infection (A. J. Lee et al., 2017), and previously thought to be important for

383 orchestrating a protective innate immune response. Accordingly, mice deficient in IL-18 are more

384 susceptible to HSV-2 infection (Harandi et al., 2001), as well as infection with HSV-1 through

385 multiple routes of inoculation (Fujioka et al., 1999; Reading et al., 2007), presumably due to

386 dysregulation of innate IFN $\gamma$ production and loss of viral control. Our study reveals a novel aspect

387 of IL-18 biology during HSV-2 infection, and that like type I IFN signaling, there may be a

388 temporal component to the effects of IL-18 during HSV-2 infection. Therapeutic neutralization of

389 IL-18 did not alter viral titers in our model, suggesting that IL-18 does not have an impact on T

390 cell-dependent IFN $\gamma$ production (Milligan \& Bernstein, 1997; Nakanishi, Lu, Gerard, \& Iwasaki,

391 2009) or direct antiviral activity. As previous studies have shown that IL-18 is also dispensable for

392 stimulating IFN $\gamma$ from adaptive memory immune responses (Harandi et al., 2001), IL-18 may be

393 an attractive target for therapeutics aiming to reduce inflammation during genital herpes.

394 Currently, the mechanism by which IL-18 promotes disease during genital HSV-2 infection is

395 unknown. In the gut, the role of IL-18 is balanced between protection and pathology, depending

396 on the source of IL-18, the model of disease and the responsive cell type (Jarret et al., 2020;

397 Nowarski et al., 2015). The role of IL-18 during HSV-2 infection appears to be similarly complex,

398 and further study will be required to identify the compartment on which IL-18 acts and the

399 downstream effects of IL-18 signaling. Additionally, while our results demonstrate an important 
400 role for IL-18, the reduction in disease severity is not as profound as when type I IFN signaling is

401 inhibited in our HSV-2 model of infection. Considering the complex response elicited by type I

402 IFN, our data allude to the possibility of other IL-18-independent, IFN-dependent mechanisms that

403 promote genital inflammation that have yet to be elucidated.

405 maximizing cellular responses to infection through the upregulation of cooperative or independent

406 molecular programs (Bartee \& McFadden, 2013) or through the cross-regulation of receptor

407 signaling pathways (Ivashkiv \& Donlin, 2014). Along with sustained type I IFN signaling during

408 HSV-2, the vaginal cytokine milieu also changes during the course of genital HSV-2 infection.

409 Whether the ISG profile induced in neutrophils by type I IFN signaling changes over the course

410 of acute HSV-2 infection, and whether this profile is affected by other cytokines, is unknown.

411 Upon activation, the activity of neutrophils responding to infection can be modulated strongly by

412 multiple interferons (IFNs), in a variety of tissues. Type I (Ank et al., 2008), type II (Iijima et al.,

413 2008; A. G. Lee et al., 2020) and type III IFNs (Ank et al., 2008; A. G. Lee et al., 2020) are all

414 robustly produced during HSV-2 infection. While type I and type II IFNs are crucial for control of

415 HSV-2 replication, endogenous type III IFNs do not appear to affect either disease severity or viral

416 control, although exogenous application of type III IFNs can reduce viral burden (Ank et al., 2008;

417 Ank et al., 2006). Expression of the type III IFN receptor, IFNLR, is limited to very few cell types,

418 including neutrophils and epithelial cells (Blazek et al., 2015; Mahlakõiv, Hernandez, Gronke,

419 Diefenbach, \& Staeheli, 2015; Sommereyns, Paul, Staeheli, \& Michiels, 2008). As epithelial cells

420 are a major target for HSV-2 replication, dissecting the action of type III IFNs within the neutrophil

421 and epithelial cell compartments may reveal a more detailed picture of the role type III IFNs play.

422 The impact of simultaneous type I, II and III IFN signaling on neutrophil function is currently 
423 unclear and due to the importance of these molecules, as well as PRRs, in controlling infection,

424 cell-specific modifications of receptor expression will be required for further investigation.

425 Beyond their inflammatory role during HSV-2 infection, this study and others (S. Li et al.,

426 2018; Milligan, 1999) have demonstrated that neutrophils can be found in the vagina at steady

427 state. Detection of neutrophils in washes collected from the vaginal lumen suggest that the

428 neutrophils are actively extravasating, migrating through the lamina propria and through the

429 epithelial barrier. Previous studies have indicated that in mice, recruitment of neutrophils into the

430 vagina is regulated by hormone-dependent expression of chemokines, including CXCR2 ligands,

431 in the tissue (Lasarte et al., 2016). In humans, the number of neutrophils in the fluctuates with the

432 hormone cycle in the upper reproductive tract, but reportedly remains stable in the lower tract

433 (Wira, Rodriguez-Garcia, \& Patel, 2015). It is unclear why neutrophils actively patrol the vagina

434 even in the absence of infection, what consequences their immunosurveillance has on the normal

435 physiology of the reproductive tract, and how the tissue microenvironment affects the biology of

436 the neutrophils themselves. In the oral mucosa, constant recruitment of neutrophils into the tissue

437 helps support the maintenance of a healthy microbiome (Uriarte, Edmisson, \& Jimenez-Flores,

438 2016), while molecular products derived from the microbiome such as peptidoglycan can support

439 basal activity of neutrophils (Clarke et al., 2010). Whether similar interactions between the vaginal

440 neutrophils and the vaginal microbiome also occur is unknown. Furthermore, due to the lack of

441 tractable animal models, the role of neutrophils in other common non-viral STIs is unclear,

442 although neutrophils appear to play a similar pathological role in the upper reproductive tract after

443 Chlamydia infection (Lijek, Helble, Olive, Seiger, \& Starnbach, 2018). In summary, our study

444 reveals that pathology during genital HSV-2 infection is driven at least in part by a robust 
bioRxiv preprint doi: https://doi.org/10.1101/2020.12.20.423690; this version posted December 23, 2020. The copyright holder for this preprint (which was not certified by peer review) is the author/funder, who has granted bioRxiv a license to display the preprint in perpetuity. It is made available under aCC-BY 4.0 International license.

445 neutrophil response, and lays the foundation for identifying the host pathways that may be targeted

446 to help ameliorate disease. 


\section{ACKNOWLEDGEMENTS}

449 We thank Rachel Idol, Antonina Akk and Celeste Cummings for technical assistance on assays

450 used in this study. This work was supported by grants from the NIH (HS: R01 AI134962) and

451 Children's Discovery Institute (RAC). T.J.L was supported by funding for the Training Program

452 in Immunology from the NIH (T32 AI007163).

\section{Author contributions}

455 T.J.L, Y.S.L., A.C., M.R.F., J.M.S., A.N.O., X.J., and H.S. designed and conducted experiments, 456 acquired data and analyzed data. R.A.C., C.T.N.P, and M.C.D. aided in the design of experiments.

457 P.S.A. and M.A. analyzed data. H.S. and Y.S.L. wrote the manuscript, and all authors edited the 458 final version of the paper.

\section{Declaration of Interests}

461 The authors declare no competing interests. 


\section{FIGURE SUPPLEMENTS}

463 Figure 1 - Supplement 1 . Neutrophil depletion does not affect magnitude of the immune cell

464 response after HSV-2 infection.

465 Figure 1 - Supplement 2. PAD4 is not required for development of genital inflammation during 466 HSV-2 infection.

467 Figure 1 - Supplement 3. ROS production and STIM1/STIM2 expression in neutrophils are not 468 required for genital inflammation after HSV-2 infection

469 Figure 2 - Supplement 1. Neutrophil depletion prior to HSV-1 genital infection has little impact 470 on disease progression.

471 Figure 3 - Supplement 1. Validation of ISG expression in the vagina.

472 Figure 3 - Supplement 3. Type I IFN is robustly produced in the vagina early after acute HSV-1 473 or HSV-2 infection.

474 Figure 4 - Supplement 1. Early blockade of IFNAR1 leads to accelerated and more severe disease 475 after HSV-2 infection.

476 Figure 6 - Supplement 1. Neutrophils do not control production of common pro-inflammatory and 477 antiviral cytokines during HSV-2 infection.

\section{SOURCE DATA}

480 Source data files have been provided for all figures and figure supplements. 


\section{METHODS}

Mice. Six-week old female C57BL/6J mice were purchased from Jackson Laboratories and rested for at least one week and infected at a minimum of seven weeks of age. Ncf2 KO mice and controls were provided by M.C. Dinauer (Washington University, St Louis) and generated as previously described (Jacob et al., 2017). Stim $1^{\mathrm{fl} / \mathrm{fl}} \times \mathrm{Stim} 2^{\mathrm{fl} / \mathrm{fl}}$ x MRP8-Cre mice were provided by

G.A. Clemens (Washington University, St Louis) and were generated as previously described 488 (Clemens et al., 2017). IFNAR1 $1^{\mathrm{fl} / \mathrm{fl}}$ mice (Ifnar $1^{\mathrm{tm} 1 \mathrm{Uka}}$ ) were a gift from H.W. Virgin (Kamphuis, 489 Junt, Waibler, Forster, \& Kalinke, 2006; Nice et al., 2016). PAD4 ${ }^{\mathrm{fl} / \mathrm{fl}}$ mice (B6(Cg)$\left.490 \mathrm{Padi}^{\text {tm1.2Kmow }} / \mathrm{J}\right)$ and MRP8-Cre (B6.Cg-Tg(S100A8-cre,-EGFP)1Ilw/J) were obtained from 491 Jackson Laboratories and bred at Washington University School of Medicine. Cre- littermates 492 generated from breeding pairs were used as controls. All mice were maintained on a 12 hour 493 light/dark cycle with unlimited access to food and water. This study was carried out in accordance 494 with the recommendations in the Guide for the Car and Use of Laboratory Animals of the National 495 Institutes of Health.

Ethics statement. The protocols were approved by the Institutional Animal Care and use

497 Committee (IACUC) at the Washington University School of Medicine (Assurance number 498 A3381-01). All experiments were performed under biosafety level 2 (A-BSL2) containment and 499 all efforts were made to minimize animal suffering.

Cell lines and primary cells. Vero Cells (African green monkey kidney epithelial cells,

501 ATCC) were cultured in Dulbeco's Modified Eagle Medium (Gibco) containing 1\% fetal bovine 502 serum (FBS, Corning) and maintained at $37^{\circ} \mathrm{C}$ with $5 \% \mathrm{CO}_{2}$. Primary neutrophils were isolated 503 from the bone marrow (BM) of naive female $\mathrm{C} 57 \mathrm{BL} / 6 \mathrm{~J}$ mice. A Histopaque gradient was used to 504 isolate primary neutrophils for Reactive Oxygen Species (ROS) assays, while a Percoll gradient 
was used for NET assays. For Histopaque isolation: 3ml of Histopaque 1119 (Sigma-Aldrich) was

overlaid with $3 \mathrm{ml}$ of Histopaque 1077 (Sigma-Aldrich). A single cell suspension of isolated BM cells in $1 \mathrm{ml}$ of PBS was layered over the Histopaque gradient. Cells were centrifuged for 30 minutes at room temperature (RT), and neutrophils were collected from the bottom interface. For Percoll isolation: BM cells were resuspended in HBSS (Gibco) with 20mM HEPES (Gibco) and

510 layered over $6 \mathrm{ml}$ of $62 \%$ Percoll solution (GE Healthcare). Cells were centrifuged for 30 minutes

511 at RT, and neutrophils were collected from the bottom of the tube. All tissue culture experiments

512 were performed under BSL2 containment.

Viruses and virus quantification. WT HSV-2 186 syn+ (Spang, Godowski, \& Knipe,

514 1983) and HSV-1 McKrae (Williams, Nesburn, \& Kaufman, 1965) was propagated and titered on

515 Vero cells as previously described (A. G. Lee et al., 2020). Briefly, for propagation of virus stocks,

516 Vero cells were plated in T150 tissue culture flasks, inoculated at $0.01 \mathrm{MOI}$ at $80 \%$ confluence

517 and incubated at $37^{\circ} \mathrm{C}$. Infected cells were harvested 2-3 days after infection, resuspended in equal

518 volumes of virus supernatant and twice-autoclaved milk, and sonicated. Lysed cells were aliquoted

519 and used as viral stock. To titer, Vero cells were plated in 6-well plates and inoculated with 10-

520 fold serial dilutions of stock virus. After inoculation, overlay media with $20 \mu \mathrm{g} / \mathrm{ml}$ human IgG was

521 added to each well and plates were incubated at $37^{\circ} \mathrm{C}$ for $2-3$ days. To count, Vero cells were

522 stained with $0.1 \%$ crystal violet. All tissue culture experiments were performed under BSL2

523 containment. For titration of virus in the vaginal lumen, 50ul washes with sterile PBS were

524 collected using a pipette and a sterile calginate swab, and diluted in 950ul of ABC buffer $(0.5 \mathrm{mM}$

$525 \mathrm{CaCl}_{2}, 0.5 \mathrm{mM} \mathrm{MgCl} 2,1 \%$ glucose, $1 \%$ fetal bovine serum (FBS) in sterile PBS). 10-fold serial

526 dilution of vaginal washes were titered by plaque assays won Vero cells (A. G. Lee et al., 2020). 
Mouse infection studies. All mice were in injected subcutaneously in the neck ruff once with 2mg of DMPA (Depo-Provera, Pfizer) 5-7 days prior to virus inoculation. For experiments in which neutrophils were depleted, mice were intraperitoneally (i.p.) injected once with $500 \mu \mathrm{g}$ of anti-Ly6G (clone 1A8) or rat IgG2a isotype control (anti-trinitrophenol+KLH) (Leinco

531 Technologies) diluted in sterile phosphate buffered saline (PBS, Sigma-Aldrich) 1 day prior to 532 inoculation. For experiments in which IFNAR blockade was conducted, mice were i.p. injected 533 once with 1mg of anti-IFNAR1 (clone MAR1-5A3) or mouse IgG1 isotype control (clone HKSP) 534 (Leinco Technologies) on either the day of inoculation ("early") or at 4 d.p.i. ("late"). For "late" 535 treatments, only mice without overt signs of genital inflammation were chosen for antibody 536 injection in both anti-IFNAR and isotype control groups to avoid biasing of results. For 537 experiments in which IL-18 was neutralized, mice were treated intravaginally with $100 \mu \mathrm{g}$ of anti538 IL-18 antibody (clone YIGIF74-1G7) or rat IgG2a isotype control (clone 2A3) (BioXCell) on days 3-5 after infection. Selection of mice for isotype control or experimental antibody treatment was random. For intravaginal inoculation, a sterile calginate swab (McKesson) moistened with sterile

541 PBS was used to gently disrupt mucous from the vaginal cavity. Stock virus was diluted in sterile

542 PBS and either 5000 PFU or $10^{4}$ PFU virus was delivered into the vaginal cavity via pipette tip in

543 a $10 \mu 1$ volume. Mice were weighed and monitored for signs of disease for 1 week following 544 infection and monitored for survival for 2 weeks. Genital inflammation was scored as follows: 0 545 no inflammation, 1 - mild redness and swelling around the vaginal opening, 2 - fur loss and visible 546 ulceration, 3 - severe ulceration and mild signs of sickness behavior (lack of grooming), 4 547 hindlimb paralysis, and 5 - moribund.

Vaginal tissue processing. All tissues were harvested from animals sedated with ketamine 
550 as follows: tissue was cut into pieces and digested for 15 minutes in a shaking water bath held at

$55137^{\circ} \mathrm{C}$ in a $0.5 \mathrm{mg} / \mathrm{ml}$ solution of Dispase II (Roche) in PBS. Tissues were then transferred to a

552 solution of $0.5 \mathrm{mg} / \mathrm{ml}$ Collagenase D (Roche) and $15 \mu \mathrm{g} / \mathrm{ml}$ DNase I (Roche) in RPMI media

553 (Gibco) supplemented with 10\% fetal bovine serum (FBS, Corning) and 1\% pen/strep (Gibco) and

554 digested for 25 minutes in a shaking water bath held at $37^{\circ} \mathrm{C} .50 \mu \mathrm{l}$ of sterile EDTA was added to

555 each sample and incubated at $37^{\circ} \mathrm{C}$ for another 5 minutes. Tissue were then mechanically disrupted

556 through a 70-um cell strainer into a single cell suspension using a $3 \mathrm{ml}$ syringe plunger. Tissues

557 were washed with RPMI media with $1 \%$ FBS, centrifuged, and resuspended in $200 \mu 1$ RPRM with

$558 \quad 1 \%$ FBS and $1 \%$ pen/strep.

Flow cytometry. Single cell suspensions from vaginal tissues, or luminal cells collected in

560 vaginal washes were plated in 96-well plates and incubated with Live/Dead Fixable Aqua Dead

561 Cell Stain kit (Molecular Probes) for 15 minutes at room temperature (RT) in the dark. Cells were

562 then incubated with Fc block (anti-CD16/32, Biolegend) for 15 minutes at RT in the dark. Surface

563 staining was performed in FACS buffer (1\% FBS and $0.02 \%$ sodium azide in PBS) on ice and in

564 the dark using the following antibodies: CD3 (clone 145-2C11), CD4 (clone GK1.5), CD8a (clone

565 53-6.7), CD11b (clone M1/70), CD45 (clone 30-F11), Gr-1 (clone RB6-8C5), Ly6C (clone

566 HK1.4), Ly6G (clone 1A8), and NK1.1 (clone PK136). All antibodies were purchased from

567 Biolegend. For surface staining of IFNAR1, cells were incubated with an anti-IFNAR1 antibody

568 or a mouse IgG1 isotype control (Leinco Technologies) for 20 minutes at $37^{\circ} \mathrm{C}$. Cells were washed

569 and then surface staining of other markers proceeded as described above. Cell counts were

570 performed by adding Precision Count Beads (Biolegend) to samples prior to flow cytometric

571 acquisition. Samples were acquired on an LSR Fortessa (BD Biosciences) and analyzed by FlowJo

572 (Treestar). 

minimum of $15 \mathrm{ml}$ of PBS, followed by $15 \mathrm{ml}$ of PLP fixative $(0.01 \mathrm{M} \mathrm{NaIO}, 0.075 \mathrm{M}$ lysine, 0.0375M sodium phosphate, 2\% paraformaldehyde (PFA)) for IF or 4\% PFA for IHC. Tissues were cryoprotected in 30\% sucrose, frozen in OCT medium (Sakura) and cut into 7um sections.

578 Cryosections were blocked 5\% bovine serum albumin (BSA), 5\% goat serum (Jackson Immunoresearch) and $0.1 \%$ Triton-X in PBS for 1 hour at RT. HSV antigens were detected with a rabbit anti-HSV primary antibody (Dako), incubated overnight at $4{ }^{\circ} \mathrm{C}$, washed in $\mathrm{PBS}$ and incubated for 1 hour at RT with a goat anti-rabbit IgG conjugated to AlexaFluor 488 (Life Technologies). S100A8 was detected with a rat anti-mouse S100A8 primary antibody (clone 583 63N13G5, Novus Biologicals) and a goat anti-rat IgG conjugated to AlexaFluor 568 (Life 584 Technologies) in a similar manner. IL-18 was detected using a biotinylated rat anti-mouse IL-18 585 primary antibody (clone 93-10C, MBL International). Cryosections were blocked as described above, and then treated with the Avidin/Biotin Blocking Kit (Vector Laboratories) according to manufacturer's protocol. Endogenous peroxidases were quenched with a $2 \%$ hydrogen peroxide 588 solution. Anti-mouse IL-18 or a rat IgG1 isotype control were incubated overnight at $4^{\circ} \mathrm{C}$. The AlexaFluor 647 Tyramide Signal Amplification kit (Invitrogen) was used to visualize IL-18 and 590 used according to manufacturer's protocol. DNA was visualized with 4',6-diamidino-2591 phenylindole (DAPI) (Life Technologies). Sections were imaged with a Zeiss Cell Observer 592 inverted microscope using a 40x objective, acquired with Zen software and image brightness was 593 adjusted using Photoshop (Adobe). For IHC, sections were probed with an anti-HSV antibody 594 incubated overnight at $4^{\circ} \mathrm{C}$ (Dako), a donkey anti-rabbit IgG-HRP antibody (Jackson 595 Immunoresearch) for $1 \mathrm{hr}$ at RT and then enzymatically visualized by 3,3'-diaminobenzidine 
(DAB) enzyme reaction (Sigma-Aldrich). Sections were counterstained with hematoxylin and eosin and images were captured using Zeiss ZEN software on a Zeiss Cell Observer inverted microscope with an Axiocam dual B/W and color camera with a 20x objective. Image brightness was adjusted using Photoshop (Adobe) and merged with Image J64 (NIH).

601 homogenized in RLT buffer (RNeasy Kit, Qiagen) with approximately $100 \mu$ of sterile $1.0 \mathrm{~mm}$ 602 zirconia/silica beads (Biospec Products) in a bead beater. Homogenized tissue samples were 603 processed according to manufacturer's protocol using the RNeasy Mini Kit (Qiagen) and RNA 604 quality and quantity was assessed on a Nanodrop (ThermoFisher). qRT-PCR was performed in $60510 \mu \mathrm{l}$ reactions using the iTaq Universal SYBR Green One-Step kit (Biorad) according to manufacturer's protocol.

Single cell RNA-sequencing preparation. Single cell suspensions from digested vaginas at room temperature (RT) in the dark. Live cells were sorted on BD FacsAria II housed in a BSL2

611 RNase inhibitor at a concentration of 800-1400 cells/ $\mu 1$, submitted to McDonnell Genome Institute 612 and prepared for droplet-based 3' end single-cell RNA sequencing using the Chromium 3' v3 single 613 cell reagent kit per manufacturer's protocol (10x Genomics). Library sequencing was performed 614 on a NovaSeq S4 (Illumina).

615 Cytokine measurement. For cytokine analysis by Bio-Plex Pro Mouse Cytokine 23-Plex

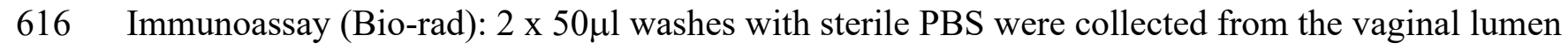
617 using a pipette. Samples were centrifuged for 3 minutes at $13000^{*} \mathrm{~g}$ to remove mucous and cells, 618 and supernatants were added to $200 \mu \mathrm{l}$ of $\mathrm{ABC}$ buffer. The assay was performed according to 
619 manufacturer instructions, and plates were read on a Luminex Bioplex 100 system (Biorad). For

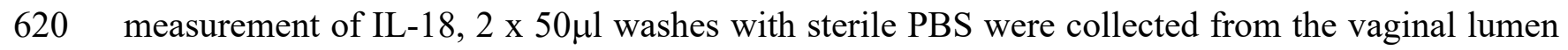

621 and centrifuged to remove mucous and cells. IL-18 was measured using the mouse IL-18 ELISA

622 kit (MBL International) according to manufacturer's instructions at half-volumes.

In vitro neutrophil stimulation. To measure ROS production, isolated neutrophils were

624 stimulated with heat-killed $\mathrm{HSV}-2\left(56^{\circ} \mathrm{C}\right.$ for 30 minutes $)$ at an MOI of 5 for 16 hours at $37^{\circ} \mathrm{C}$.

625 ROS levels were quantified using DCFDA Cellular ROS Detection Assay kit (Abcam) according

626 to manufacturer protocol. Fluorescence levels were measured by flow cytometry. To induce NET

627 formation, neutrophils were stimulated with heat-killed HSV-2 at an MOI if 1 for 4 hours at $37^{\circ} \mathrm{C}$.

628 Cells were fixed with 8\% PFA overnight and probed with a polyclonal rabbit antibody against 629 mouse citrullinated histone $\mathrm{H} 3$ (Abcam) for 1 hour at RT in 1\% BSA and 0.1\% Triton-X for 1

630 hour at RT, a goat anti-rabbit antibody conjugated to AlexaFluor 488 (Life Technologies) for 1

631 hour at RT and DAPI diluted in PBS for 6 minutes at RT. Cells were imaged with a Zeiss Cell

632 Observer inverted microscope using a 63x objective and image brightness was adjusted using

633 Photoshop (Adobe).

634 Single cell RNA-sequencing analysis.

635 Processing data with Seurat package: The Seurat package in $\mathrm{R}$ was used for analysis

636 (Butler, Hoffman, Smibert, Papalexi, \& Satija, 2018). Cells with mitochondrial content greater

637 than 5\% were removed. The initial analysis of the data revealed three clusters of the cells that had

638 extremely low levels of detected genes (i.e. $<500)$ which were then filtered out as non-viable cells.

639 Remaining cells were used for downstream analysis, resulting in the 6,507 cells per sample that

640 passed quality control (QC) and filtering. Filtered data were normalized using a scaling factor of

641 10,000, and nUMI was regressed with a negative binomial model. 
643 a scaling factor of 10,000 and $\log$ transformed. The highly variable genes were selected using the

644 FindVariableFeatures function with mean greater than 0.0125 or less than 3 and dispersion greater

645 than 0.5 . These genes were used in performing the linear dimensionality reduction.

647 top 3000 most variable genes prior to clustering and number of the first principal components

648 (PCs) were used based on the ElbowPlot as described below for different datasets. Clustering was

649 performed using the FindClusters function which works on K-nearest neighbor (KNN) graph

650 model with the granularity (resolution) ranging from $0.1-1.5$. The datasets were projected as t-SNE

651 plots.

Statistical analysis. All numerical data analysis except for scRNA-seq data analysis was

653 performed on Graphpad Prism8 software. Values were log-transformed to normalize distribution

654 and variances where necessary. Immune cell numbers and cytokine measurement were analyzed

655 by 2-way ANOVA with Bonferroni multiple comparisons test. Log-transformed viral titers were

656 analyzed by repeated measures two-way ANOVA with Bonferroni multiple comparisons test.

657 Inflammation scores were analyzed by repeated-measures two-way ANOVA or mixed-effects

658 analysis with Geisser-Greenhouse correction and Bonferroni's multiple comparisons test. The

659 Geisser-Greenhouse correction was used for inflammation scores to correct any violations of 660 sphericity and to provide a more restrictive, stringent calculation of $p$ values. ROS MFI was

661 measured by unpaired two-tailed Student's t-test. qPCR results were analyzed by one-way 662 ANOVA with Tukey's multiple comparisons test. A $p<0.05$ was considered statistically 663 significant. No experimental data points were excluded from statistical analysis, including 664 potential outliers. Mouse and sample numbers per group and experimental repeat information is 
665 provided in the figure legends. All data points represent individual biological replicates, and the

666 ' $\mathrm{n}$ ' for each group refers to biological replicates. No power calculations were performed to

667 determine sample size; rather sample sizes were determined based on historical data. 
bioRxiv preprint doi: https://doi.org/10.1101/2020.12.20.423690; this version posted December 23, 2020. The copyright holder for this preprint

(which was not certified by peer review) is the author/funder, who has granted bioRxiv a license to display the preprint in perpetuity. It is made available under aCC-BY 4.0 International license.

\section{DATA AVAILABILITY}

669 The published article includes partial data from a single cell RNA-sequencing dataset generated

670 during this study. These data are available at Gene Expression Omnibus (GEO) (accession code

671 GSE161336). The key to access this data is sngheyymjxqbjsn.

672 


\section{REFERENCES}

Akk, A., Springer, L. E., \& Pham, C. T. N. (2016). Neutrophil Extracellular Traps Enhance Early Inflammatory Response in Sendai Virus-Induced Asthma Phenotype. Frontiers in Immunology, 7, 325. doi:10.3389/fimmu.2016.00325

Ank, N., Iversen, M. B., Bartholdy, C., Staeheli, P., Hartmann, R., Jensen, U. B., . . Paludan, S. R. (2008). An Important Role for Type III Interferon (IFN- $\lambda /$ IL-28) in TLR-Induced Antiviral Activity. The Journal of Immunology, 180(4), 2474.

Ank, N., West, H., Bartholdy, C., Eriksson, K., Thomsen, A. R., \& Paludan, S. R. (2006). Lambda interferon (IFN-lambda), a type III IFN, is induced by viruses and IFNs and displays potent antiviral activity against select virus infections in vivo. Journal of Virology, 80(9), 45014509. doi:10.1128/JVI.80.9.4501-4509.2006

Bai, F., Kong, K.-F., Dai, J., Qian, F., Zhang, L., Brown, C. R., . . Montgomery, R. R. (2010). A paradoxical role for neutrophils in the pathogenesis of West Nile virus. The Journal of Infectious Diseases, 202(12), 1804-1812. doi:10.1086/657416

Bartee, E., \& McFadden, G. (2013). Cytokine synergy: An underappreciated contributor to innate anti-viral immunity.

Cytokine,

63(3), $237-240$. doi:https://doi.org/10.1016/j.cyto.2013.04.036

Blazek, K., Eames, H. L., Weiss, M., Byrne, A. J., Perocheau, D., Pease, J. E., . . Udalova, I. A. (2015). IFN- $\lambda$ resolves inflammation via suppression of neutrophil infiltration and IL-1 $\beta$ production. The Journal of Experimental Medicine, 212(6), 845-853. doi:10.1084/jem.20140995 
695 Boddingius, J., Dijkman, H., Hendriksen, E., Schift, R., \& Stolz, E. (1987). HSV-2 replication sites, monocyte and lymphocytic cell infection and virion phagocytosis by neutrophils, in vesicular lesions on penile skin. Journal of Cutaneous Pathology, 14(3), 165-175. doi:10.1111/j.1600-0560.1987.tb00492.x

Brandes, M., Klauschen, F., Kuchen, S., \& Germain, R. N. (2013). A systems analysis identifies a feedforward inflammatory circuit leading to lethal influenza infection. Cell, 154(1), 197-

Butler, A., Hoffman, P., Smibert, P., Papalexi, E., \& Satija, R. (2018). Integrating single-cell transcriptomic data across different conditions, technologies, and species. Nature Biotechnology, 36(5), 411-420. doi:10.1038/nbt.4096

Cheng, L., Yu, H., Li, G., Li, F., Ma, J., Li, J., . . Su, L. (2017). Type I interferons suppress viral replication but contribute to $\mathrm{T}$ cell depletion and dysfunction during chronic HIV-1 infection. JCI Insight, 2(12). doi:10.1172/jci.insight.94366

Christensen, M. H., Jensen, S. B., Miettinen, J. J., Luecke, S., Prabakaran, T., Reinert, L. S., . . .

Clancy, D. M., Sullivan, G. P., Moran, H. B. T., Henry, C. M., Reeves, E. P., McElvaney, N. G., . Paludan, S. R. (2016). HSV-1 ICP27 targets the TBK1-activated STING signalsome to inhibit virus-induced type I IFN expression. The EMBO journal, 35(13), 1385-1399. doi:10.15252/embj.201593458 
immunity.

Nat

Med,

$16(2)$

$228-231$. doi:http://www.nature.com/nm/journal/v16/n2/suppinfo/nm.2087_S1.htm1

Clemens, R. A., Chong, J., Grimes, D., Hu, Y., \& Lowell, C. A. (2017). STIM1 and STIM2 cooperatively regulate mouse neutrophil store-operated calcium entry and cytokine production.

Blood, 130(13), 1565.

Retrieved

from

\section{4}

725

726

727

728

729

730

731

732

733

734

735

736

737

738

739 http://www.bloodjournal.org/content/130/13/1565.abstract

Dinauer, M. C. (2019). Inflammatory consequences of inherited disorders affecting neutrophil function. Blood, 133(20), 2130-2139. doi:10.1182/blood-2018-11-844563

Divito, S. J., \& Hendricks, R. L. (2008). Activated inflammatory infiltrate in HSV-1-infected corneas without herpes stromal keratitis. Investigative ophthalmology \& visual science, 49(4), 1488-1495. doi:10.1167/iovs.07-1107

Fujioka, N., Akazawa, R., Ohashi, K., Fujii, M., Ikeda, M., \& Kurimoto, M. (1999). Interleukin18 Protects Mice against Acute Herpes Simplex Virus Type 1 Infection. Journal of Virology, 73(3), 2401-2409. Retrieved from http://jvi.asm.org/content/73/3/2401.abstract

Galani, I. E., \& Andreakos, E. (2015). Neutrophils in viral infections: Current concepts and caveats. Journal of Leukocyte Biology, 98(4), 557-564. doi:10.1189/jlb.4VMR1114-555R

Galani, I. E., Triantafyllia, V., Eleminiadou, E.-E., Koltsida, O., Stavropoulos, A., Manioudaki, M., ... Andreakos, E. (2017). Interferon- $\lambda$ Mediates Non-redundant Front-Line Antiviral Protection against Influenza Virus Infection without Compromising Host Fitness. Immunity, 46(5), 875-890.e876. doi:https://doi.org/10.1016/j.immuni.2017.04.025

Gill, N., Deacon, P. M., Lichty, B., Mossman, K. L., \& Ashkar, A. A. (2006). Induction of Innate Immunity against Herpes Simplex Virus Type 2 Infection via Local Delivery of Toll-Like 
Receptor Ligands Correlates with Beta Interferon Production. Journal of Virology, 80(20), 9943-9950. doi:10.1128/jvi.01036-06

742 Gopinath, S., Kim, M. V., Rakib, T., Wong, P. W., van Zandt, M., Barry, N. A., . . Iwasaki, A. (2018). Topical application of aminoglycoside antibiotics enhances host resistance to viral infections in a microbiota-independent manner. Nature Microbiology, 3(5), 611-621.

Gosmann, C., Anahtar, M. N., Handley, S. A., Farcasanu, M., Abu-Ali, G., Bowman, B. A., . . . Kwon, D. S. (2017). Lactobacillus-Deficient Cervicovaginal Bacterial Communities Are Associated with Increased HIV Acquisition in Young South African Women. Immunity,

Granger, V., Peyneau, M., Chollet-Martin, S., \& de Chaisemartin, L. (2019). Neutrophil Extracellular Traps in Autoimmunity and Allergy: Immune Complexes at Work. Frontiers in Immunology, 10(2824). doi:10.3389/fimmu.2019.02824

753 Gupta, S., Nakabo, S., Blanco, L. P., O’Neil, L. J., Wigerblad, G., Goel, R. R., . . Kaplan, M. J. (2020). Sex differences in neutrophil biology modulate response to type I interferons and immunometabolism. Proceedings of the National Academy of Sciences, 117(28), 1648116491. doi:10.1073/pnas.2003603117

757 Harandi, A. M., Svennerholm, B., Holmgren, J., \& Eriksson, K. (2001). Interleukin-12 (IL-12) and IL-18 are important in innate defense against genital herpes simplex virus type 2 infection in mice but are not required for the development of acquired gamma interferon-mediated protective immunity. Journal of Virology, 75(14), 6705-6709. doi:10.1128/JVI.75.14.6705-6709.2001 
762 Harris, L. D., Tabb, B., Sodora, D. L., Paiardini, M., Klatt, N. R., Douek, D. C., . . Estes, J. D. (2010). Downregulation of Robust Acute Type I Interferon Responses Distinguishes Nonpathogenic Simian Immunodeficiency Virus (SIV) Infection of Natural Hosts from Pathogenic SIV Infection of Rhesus Macaques. Journal of Virology, 84(15), 7886-7891. doi:10.1128/jvi.02612-09

767

768

Horbul, J. E., Schmechel, S. C., Miller, B. R. L., Rice, S. A., \& Southern, P. J. (2011). Herpes Simplex Virus-Induced Epithelial Damage and Susceptibility to Human Immunodeficiency Virus Type 1 Infection in Human Cervical Organ Culture. PLoS ONE, 6(7), e22638. doi:10.1371/journal.pone.0022638

Iijima, N., Linehan, M. M., Zamora, M., Butkus, D., Dunn, R., Kehry, M. R., . . Iwasaki, A. (2008). Dendritic cells and B cells maximize mucosal Th1 memory response to herpes simplex virus. The Journal of Experimental Medicine, 205(13), 3041-3052. doi:10.1084/jem.20082039

Iijima, N., Mattei, L. M., \& Iwasaki, A. (2011). Recruited inflammatory monocytes stimulate antiviral Th1 immunity in infected tissue. Proceedings of the National Academy of Sciences of the United States of America, 108(1), 284-289. doi:10.1073/pnas.1005201108

Ivashkiv, L. B., \& Donlin, L. T. (2014). Regulation of type I interferon responses. Nature reviews. Immunology, 14(1), 36-49. doi:10.1038/nri3581

Iversen, M. B., Ank, N., Melchjorsen, J., \& Paludan, S. R. (2010). Expression of type III interferon (IFN) in the vaginal mucosa is mediated primarily by dendritic cells and displays stronger dependence on NF-kappaB than type I IFNs. Journal of Virology, 84(9), 4579-4586. doi:10.1128/JVI.02591-09 
Iversen, M. B., Reinert, L. S., Thomsen, M. K., Bagdonaite, I., Nandakumar, R., Cheshenko, N., . . Paludan, S. R. (2015). An innate antiviral pathway acting before interferons at epithelial surfaces. Nat Immunol, advance online publication. doi:10.1038/ni.3319 surfaces. Nature immunology, 17(2), 150-158. doi:10.1038/ni.3319

Jacob, C. O., Yu, N., Yoo, D.-G., Perez-Zapata, L. J., Barbu, E. A., Kaplan, M. J., . . Dinauer, M. C. (2017). Haploinsufficiency of NADPH Oxidase Subunit Neutrophil Cytosolic Factor 2 Is Sufficient to Accelerate Full-Blown Lupus in NZM 2328 Mice. Arthritis \&

Jacquelin, B., Mayau, V., Targat, B., Liovat, A.-S., Kunkel, D., Petitjean, G., . . Müller-Trutwin, M. C. (2009). Nonpathogenic SIV infection of African green monkeys induces a strong but

Jarret, A., Jackson, R., Duizer, C., Healy, M. E., Zhao, J., Rone, J. M., . . Flavell, R. A. (2020). Enteric Nervous System-Derived IL-18 Orchestrates Mucosal Barrier Immunity. Cell,

Jenne, C. N., \& Kubes, P. (2015). Virus-induced NETs--critical component of host defense or pathogenic mediator? PLOS Pathogens, 11(1), e1004546-e1004546. doi:10.1371/journal.ppat.1004546 180(1), 50-63.e12. doi:https://doi.org/10.1016/j.cell.2019.12.016 
from Virus Challenge by Releasing Neutrophil Extracellular Traps. Cell Host \& Microbe,

Kamphuis, E., Junt, T., Waibler, Z., Forster, R., \& Kalinke, U. (2006). Type I interferons directly regulate lymphocyte recirculation and cause transient blood lymphopenia. Blood, 108(10),

Kaushic, C., Ashkar, A. A., Reid, L. A., \& Rosenthal, K. L. (2003). Progesterone Increases

Khoury-Hanold, W., Yordy, B., Kong, P., Kong, Y., Ge, W., Szigeti-Buck, K., . . Iwasaki, A. (2016). Viral Spread to Enteric Neurons Links Genital HSV-1 Infection to Toxic

from

\section{$\underline{\text { http://jvi.asm.org/cgi/content/abstract/77/8/4558 }}$} Megacolon and Lethality. Cell Host \& Microbe, 19(6), 788-799. doi:http://dx.doi.org/10.1016/j.chom.2016.05.008

Krzyzowska, M., Baska, P., Grochowska, A., Orlowski, P., Nowak, Z., \& Winnicka, A. (2014). Fas/FasL pathway participates in resolution of mucosal inflammatory response early during HSV-2 infection. Immunobiology, 219(1), 64-77. doi:http://dx.doi.org/10.1016/j.imbio.2013.08.002

Kulkarni, U., Zemans, R. L., Smith, C. A., Wood, S. C., Deng, J. C., \& Goldstein, D. R. (2019). Excessive neutrophil levels in the lung underlie the age-associated increase in influenza mortality. Mucosal immunology, 12(2), 545-554. doi:10.1038/s41385-018-0115-3

Lasarte, S., Samaniego, R., Salinas-Muñoz, L., Guia-Gonzalez, M. A., Weiss, L. A., Mercader, E., ... Relloso, M. (2016). Sex Hormones Coordinate Neutrophil Immunity in the Vagina by 
Controlling Chemokine Gradients. Journal of Infectious Diseases, 213(3), 476-484. doi:10.1093/infdis/jiv402

831 Lee, A. G., Scott, J. M., Fabbrizi, M. R., Jiang, X., Sojka, D. K., Miller, M. J., . . Shin, H. (2020). T cell response kinetics determines neuroinfection outcomes during murine HSV infection.

Lee, A. J., \& Ashkar, A. A. (2012). Herpes simplex virus-2 in the genital mucosa: insights into the mucosal host response and vaccine development. Current Opinion in Infectious Diseases,

Lee, A. J., Chen, B., Chew, M. V., Barra, N. G., Shenouda, M. M., Nham, T., . . Ashkar, A. A. JCI Insight, 5(5). doi:10.1172/jci.insight.134258 (2017). Inflammatory monocytes require type I interferon receptor signaling to activate NK (1999). Interferons regulate the phenotype of wild-type and mutant herpes simplex viruses in vivo. The Journal of Experimental Medicine, 189(4), 663-672. Estrogen Action in the Epithelial Cells of the Mouse Vagina Regulates Neutrophil Infiltration and Vaginal Tissue Integrity. Scientific Reports, 8(1), 11247. doi:10.1038/s41598-018-29423-5 
852 Liberzon, A., Birger, C., Thorvaldsdóttir, H., Ghandi, M., Mesirov, J. P., \& Tamayo, P. (2015). 1(6), 417-425. doi:10.1016/j.cels.2015.12.004

Lijek, R. S., Helble, J. D., Olive, A. J., Seiger, K. W., \& Starnbach, M. N. (2018). Pathology after $<$ em $>$ Chlamydia trachomatis $</$ em $>$ infection is driven by nonprotective immune cells that are distinct from protective populations. Proceedings of the National Academy of Sciences,

Lin, Y., \& Zheng, C. (2019). A Tug of War: DNA-Sensing Antiviral Innate Immunity and Herpes Simplex Virus Type I Infection. Frontiers in Microbiology, 10, 2627-2627. doi:10.3389/fmicb.2019.02627

Luker, G. D., Prior, J. L., Song, J., Pica, C. M., \& Leib, D. A. (2003). Bioluminescence imaging reveals systemic dissemination of herpes simplex virus type 1 in the absence of interferon receptors. Journal of Virology, 77(20), 11082-11093. doi:10.1128/jvi.77.20.11082-

Mahlakõiv, T., Hernandez, P., Gronke, K., Diefenbach, A., \& Staeheli, P. (2015). Leukocyte- 
874 Maruzuru, Y., Ichinohe, T., Sato, R., Miyake, K., Okano, T., Suzuki, T., . . Kawaguchi, Y. (2018).

875

876

877

878

879

880

881

882

883

884

885

886

887

888

889

890

891

892

893

894

895

Herpes Simplex Virus 1 VP22 Inhibits AIM2-Dependent Inflammasome Activation to Enable Efficient Viral Replication. Cell Host \& Microbe, 23(2), 254-265.e257. doi:https://doi.org/10.1016/j.chom.2017.12.014

Masson, L., Mlisana, K., Little, F., Werner, L., Mkhize, N. N., Ronacher, K., . . Passmore, J.-A. S. (2014). Defining genital tract cytokine signatures of sexually transmitted infections and bacterial vaginosis in women at high risk of HIV infection: a cross-sectional study. Sexually transmitted infections, 90(8), 580-587. doi:10.1136/sextrans-2014-051601

Mayadas, T. N., Cullere, X., \& Lowell, C. A. (2014). The multifaceted functions of neutrophils. Annual review of pathology, 9, 181-218. doi:10.1146/annurev-pathol-020712-164023

Meier, A., Chang, J. J., Chan, E. S., Pollard, R. B., Sidhu, H. K., Kulkarni, S., . . Altfeld, M. (2009). Sex differences in the Toll-like receptor-mediated response of plasmacytoid dendritic cells to HIV-1. Nature Medicine, 15(8), 955-959. doi:10.1038/nm.2004

Melroe, G. T., DeLuca, N. A., \& Knipe, D. M. (2004). Herpes Simplex Virus 1 Has Multiple Mechanisms for Blocking Virus-Induced Interferon Production. Journal of Virology, 78(16), 8411-8420. doi:10.1128/jvi.78.16.8411-8420.2004

Milligan, G. N. (1999). Neutrophils Aid in Protection of the Vaginal Mucosae of Immune Mice against Challenge with Herpes Simplex Virus Type 2. Journal of Virology, 73(8), 63806386. Retrieved from http://www.ncbi.nlm.nih.gov/pmc/articles/PMC112717/

Milligan, G. N., \& Bernstein, D. I. (1997). Interferon- $\gamma$ Enhances Resolution of Herpes Simplex Virus Type 2 Infection of the Murine Genital Tract. Virology, 229(1), 259-268. doi:https://doi.org/10.1006/viro.1997.8441 
Milligan, G. N., Bourne, N., \& Dudley, K. L. (2001). Role of polymorphonuclear leukocytes in resolution of HSV-2 infection of the mouse vagina. Journal of Reproductive Immunology, 49(1), 49-65. doi:https://doi.org/10.1016/S0165-0378(00)00080-2

899

900

901

902

903

904

905

906

907

908

909

910

911

912

913

914

915

916

917

918

Mittal, M., Siddiqui, M. R., Tran, K., Reddy, S. P., \& Malik, A. B. (2014). Reactive Oxygen Species in Inflammation and Tissue Injury. Antioxidants \& Redox Signaling, 20(7), 11261167. doi:10.1089/ars.2012.5149

Murphy, K., \& Mitchell, C. M. (2016). The Interplay of Host Immunity, Environment and the Risk of Bacterial Vaginosis and Associated Reproductive Health Outcomes. The Journal of Infectious Diseases, 214 Suppl 1(Suppl 1), S29-S35. doi:10.1093/infdis/jiw140

Nakanishi, Y., Lu, B., Gerard, C., \& Iwasaki, A. (2009). CD8(+) T lymphocyte mobilization to virus-infected tissue requires CD4(+) T-cell help. Nature, 462(7272), 510-513. doi:10.1038/nature08511

Narasaraju, T., Yang, E., Samy, R. P., Ng, H. H., Poh, W. P., Liew, A.-A., . . Chow, V. T. (2011). Excessive neutrophils and neutrophil extracellular traps contribute to acute lung injury of influenza pneumonitis. The American Journal of Pathology, 179(1), 199-210. doi:10.1016/j.ajpath.2011.03.013

Nice, T. J., Osborne, L. C., Tomov, V. T., Artis, D., Wherry, E. J., \& Virgin, H. W. (2016). Type I Interferon Receptor Deficiency in Dendritic Cells Facilitates Systemic Murine Norovirus Persistence Despite Enhanced Adaptive Immunity. PLOS Pathogens, 12(6), e1005684e1005684. doi:10.1371/journal.ppat.1005684

Nowarski, R., Jackson, R., Gagliani, N., de Zoete, Marcel R., Palm, Noah W., Bailis, W., ... Flavell, Richard A. (2015). Epithelial IL-18 Equilibrium Controls Barrier Function in Colitis. Cell, 163(6), 1444-1456. doi:https://doi.org/10.1016/j.cell.2015.10.072 
919 Peng, T., Zhu, J., Klock, A., Phasouk, K., Huang, M.-L., Koelle, D. M., . . . Corey, L. (2009). Evasion of the Mucosal Innate Immune System by Herpes Simplex Virus Type 2. J. Virol., 83(23), 12559-12568. doi:10.1128/jvi.00939-09

922

923

924

925

926

927

928

929

930

931

932

933

934

935

936

937

938

939

Pham, C. T. N. (2006). Neutrophil serine proteases: specific regulators of inflammation. Nat Rev Immunol, 6(7), 541-550. Retrieved from http://dx.doi.org/10.1038/nri1841

Pylaeva, E., Bordbari, S., Spyra, I., Decker, A. S., Häussler, S., Vybornov, V., . . Jablonska, J. (2019). Detrimental Effect of Type I IFNs During Acute Lung Infection With Pseudomonas aeruginosa Is Mediated Through the Stimulation of Neutrophil NETosis. Frontiers in Immunology, 10(2190). doi:10.3389/fimmu.2019.02190

Rao, P., \& Suvas, S. (2018). Development of Inflammatory Hypoxia and Prevalence of Glycolytic Metabolism in Progressing Herpes Stromal Keratitis Lesions. The Journal of Immunology, ji1800422. doi:10.4049/jimmunol.1800422

Reading, P. C., Whitney, P. G., Barr, D. P., Wojtasiak, M., Mintern, J. D., Waithman, J., \& Brooks, A. G. (2007). IL-18, but not IL-12, Regulates NK Cell Activity following Intranasal Herpes Simplex Virus Type 1 Infection. The Journal of Immunology, 179(5), 3214-3221. doi:10.4049/jimmunol.179.5.3214

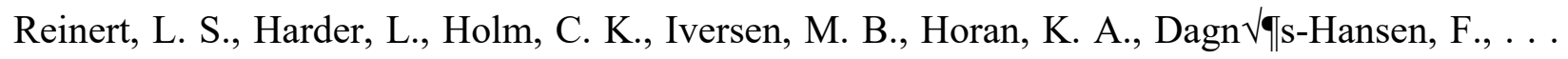
Paludan, S. r. R. (2012). TLR3 deficiency renders astrocytes permissive to herpes simplex virus infection and facilitates establishment of CNS infection in mice. The Journal of Clinical Investigation, $122(4)$ $1368-1376$.

Retrieved from http://www.jci.org/articles/view/60893 
940 Robertson, S. E., Young, J. D., Kitson, S., Pitt, A., Evans, J., Roes, J., . . McInnes, I. B. (2006). Immunology, 36(3), 722-731. doi:10.1002/eji.200535402

943 Rocha, Bruno C., Marques, Pedro E., Leoratti, Fabiana Maria de S., Junqueira, C., Pereira,

Rotger, M., Dang, K. K., Fellay, J., Heinzen, E. L., Feng, S., Descombes, P., . . Center for, H. I. Dhelio B., Antonelli, Lis Ribeiro do V., . . . Gazzinelli, Ricardo T. (2015). Type I

Roychoudhury, P., Swan, D. A., Duke, E. R., Corey, L., Zhu, J., Davé, V. A., . . Schiffer, J. T. (2020). Tissue-resident $\mathrm{T}$ cell derived cytokines eliminate herpes simplex virus-2 infected cells. The Journal of Clinical Investigation. doi:10.1172/JCI132583 Carr, D. J. J. (2019). Vaccine-induced antibodies target sequestered viral antigens to

959 Saitoh, T., Komano, J., Saitoh, Y., Misawa, T., Takahama, M., Kozaki, T., . . Akira, S. (2012).

960 Neutrophil Extracellular Traps Mediate a Host Defense Response to Human 961 Immunodeficiency Virus-1. Cell Host \& Microbe, 12(1), 109-116. 962 doi:http://dx.doi.org/10.1016/j.chom.2012.05.015 
963 Schiffer, J. T., \& Corey, L. (2013). Rapid host immune response and viral dynamics in herpes simplex virus-2 infection. Nature Medicine, 19(3), 280-290. doi:10.1038/nm.3103

965 Schiffer, J. T., Swan, D., Sallaq, R. A., Magaret, A., Johnston, C., Mark, K. E., . . . Corey, L.

966 (2013). Rapid localized spread and immunologic containment define Herpes simplex virus-

9672 reactivation in the human genital tract. eLife, 2. doi:10.7554/eLife.00288

968 Scott, J. M., Lebratti, T. J., Richner, J. M., Jiang, X., Fernandez, E., Zhao, H., . . . Shin, H. (2018). Journal of Virology, 92(7). doi:10.1128/jvi.00038-18

Sedaghat, A. R., German, J., Teslovich, T. M., Cofrancesco, J., Jie, C. C., Talbot, C. C., \& Siliciano, R. F. (2008). Chronic CD $4<$ sup $>+</$ sup $>$ T-Cell Activation and Depletion in Human Immunodeficiency Virus Type 1 Infection: Type I Interferon-Mediated Disruption

975 Shin, H., \& Iwasaki, A. (2013). Generating protective immunity against genital herpes. Trends in Immunology, 34(10), 487-494. doi:http://dx.doi.org/10.1016/j.it.2013.08.001

977 Sommereyns, C., Paul, S., Staeheli, P., \& Michiels, T. (2008). IFN-lambda (IFN-lambda) is expressed in a tissue-dependent fashion and primarily acts on epithelial cells in vivo. PLOS

980 Spang, A. E., Godowski, P. J., \& Knipe, D. M. (1983). Characterization of herpes simplex virus 2 temperature-sensitive mutants whose lesions map in or near the coding sequences for the major DNA-binding protein. Journal of Virology, 45(1), 332-342. Retrieved from 
984 Stock, A. T., Smith, J. M., \& Carbone, F. R. (2014). Type I IFN suppresses Cxcr2 driven neutrophil recruitment into the sensory ganglia during viral infection. The Journal of Experimental Medicine. doi:10.1084/jem.20132183

Sugawara, S., Uehara, A., Nochi, T., Yamaguchi, T., Ueda, H., Sugiyama, A., . . Takada, H. (2001). Neutrophil Proteinase 3-Mediated Induction of Bioactive IL-18 Secretion by Human Oral Epithelial Cells. The Journal of Immunology, 167(11), 6568-6575.

Svensson, A., Bellner, L., Magnusson, M., \& Eriksson, K. (2007). Role of IFNalpha/beta signaling in the prevention of genital herpes virus type 2 infection. Journal of Reproductive

994 Swiecki, M., Wang, Y., Gilfillan, S., \& Colonna, M. (2013). Plasmacytoid Dendritic Cells Contribute to Systemic but Not Local Antiviral Responses to HSV Infections. PLOS Immunology, 74(1,̈̈̀े2), 114-123. doi:http://dx.doi.org/10.1016/j.jri.2006.09.002

997 Taleb, K., Auffray, C., Villefroy, P., Pereira, A., Hosmalin, A., Gaudry, M., \& Le Bon, A. (2017). Chronic Type I IFN Is Sufficient To Promote Immunosuppression through Accumulation of Myeloid-Derived Suppressor Cells. The Journal of Immunology, 198(3), 1156-1163.

1001 Tate, M. D., Deng, Y.-M., Jones, J. E., Anderson, G. P., Brooks, A. G., \& Reading, P. C. (2009). Neutrophils Ameliorate Lung Injury and the Development of Severe Disease during 1003 Influenza Infection. The Journal of Immunology, 183(11), 7441-7450. doi:10.4049/jimmunol.0902497 
1005 Tate, M. D., Ioannidis, L. J., Croker, B., Brown, L. E., Brooks, A. G., \& Reading, P. C. (2011). The role of neutrophils during mild and severe influenza virus infections of mice. PLoS ONE, 6(3), e17618-e17618. doi:10.1371/journal.pone.0017618

1008 Tecchio, C., Micheletti, A., \& Cassatella, M. A. (2014). Neutrophil-derived cytokines: facts beyond expression. Frontiers in Immunology, 5, 508-508. doi:10.3389/fimmu.2014.00508

1010 Teijaro, J. R., Ng, C., Lee, A. M., Sullivan, B. M., Sheehan, K. C. F., Welch, M., . . Oldstone, M. B. A. (2013). Persistent LCMV Infection Is Controlled by Blockade of Type I Interferon Signaling. Science, 340(6129), 207-211. doi:10.1126/science.1235214

1013 Thomas, J., Gangappa, S., Kanangat, S., \& Rouse, B. T. (1997). On the essential involvement of neutrophils in the immunopathologic disease: herpetic stromal keratitis. The Journal of Immunology, $158(3)$ 1383-1391.

Retrieved from

1017 Truong, N. R., Smith, J. B., Sandgren, K. J., \& Cunningham, A. L. (2019). Mechanisms of Immune Control of Mucosal HSV Infection: A Guide to Rational Vaccine Design. Frontiers in Immunology, 10(373). doi:10.3389/fimmu.2019.00373

1020 Uriarte, S. M., Edmisson, J. S., \& Jimenez-Flores, E. (2016). Human neutrophils and oral 1021 microbiota: a constant tug-of-war between a harmonious and a discordant coexistence.

1023 Vidy, A., Maisonnasse, P., Da Costa, B., Delmas, B., Chevalier, C., \& Le Goffic, R. (2016). The 1024 Influenza Virus Protein PB1-F2 Increases Viral Pathogenesis through Neutrophil 1025 Recruitment and NK Cells Inhibition. PLoS ONE, 11(10), e0165361-e0165361. 1026 doi:10.1371/journal.pone.0165361 
1027 Wald, A., Zeh, J., Selke, S., Warren, T., Ashley, R., \& Corey, L. (2002). Genital Shedding of 1028 Herpes Simplex Virus among Men. The Journal of Infectious Diseases, 1029 186(Supplement_1), S34-S39. doi:10.1086/342969

1030 Wang, J. P., Bowen, G. N., Zhou, S., Cerny, A., Zacharia, A., Knipe, D. M., . . Kurt-Jones, E. A. (2012). Role of Specific Innate Immune Responses in Herpes Simplex Virus Infection of the Central Nervous System. Journal of Virology, 86(4), 2273-2281. doi:10.1128/jvi.06010-11

1034 Wilcox, D. R., Folmsbee, S. S., Muller, W. J., \& Longnecker, R. (2016). The Type I Interferon Response Determines Differences in Choroid Plexus Susceptibility between Newborns and Adults in Herpes Simplex Virus Encephalitis. mBio, 7(2). doi:10.1128/mBio.00437-16

Williams, L. E., Nesburn, A. B., \& Kaufman, H. E. (1965). Experimental Induction of Disciform Keratitis. $J A M A$ Ophthalmology, $73(1)$ $112-114$. doi:10.1001/archopht.1965.00970030114023

Wilson, E. B., Yamada, D. H., Elsaesser, H., Herskovitz, J., Deng, J., Cheng, G., . . Brooks, D. G. (2013). Blockade of Chronic Type I Interferon Signaling to Control Persistent LCMV Infection. Science, 340(6129), 202-207. doi:10.1126/science.1235208

Wira, C. R., Rodriguez-Garcia, M., \& Patel, M. V. (2015). The role of sex hormones in immune protection of the female reproductive tract. Nat Rev Immunol, 15(4), 217-230.

1046 World Health Organization, D. o. R. H. a. R. (2007). Global strategy for the prevention and control of sexually transmitted infections: 2006-2015. Breaking the chain of transmission.

1048 Xin, L., Vargas-Inchaustegui, D. A., Raimer, S. S., Kelly, B. C., Hu, J., Zhu, L., . . Soong, L. 
$<$ em>Leishmania</em> Parasites. The Journal of Immunology, 184(12), 7047-7056. doi:10.4049/jimmunol.0903273

1052 Zheng, G. X. Y., Terry, J. M., Belgrader, P., Ryvkin, P., Bent, Z. W., Wilson, R., . . Bielas, J. H.

1053 (2017). Massively parallel digital transcriptional profiling of single cells. Nature 1054 Communications, 8(1), 14049. doi:10.1038/ncomms14049

1055 Zhu, Q., \& Kanneganti, T.-D. (2017). Cutting Edge: Distinct Regulatory Mechanisms Control Proinflammatory Cytokines IL-18 and IL-1ß. The Journal of Immunology, 198(11), 42104215. doi:10.4049/jimmunol.1700352 
A
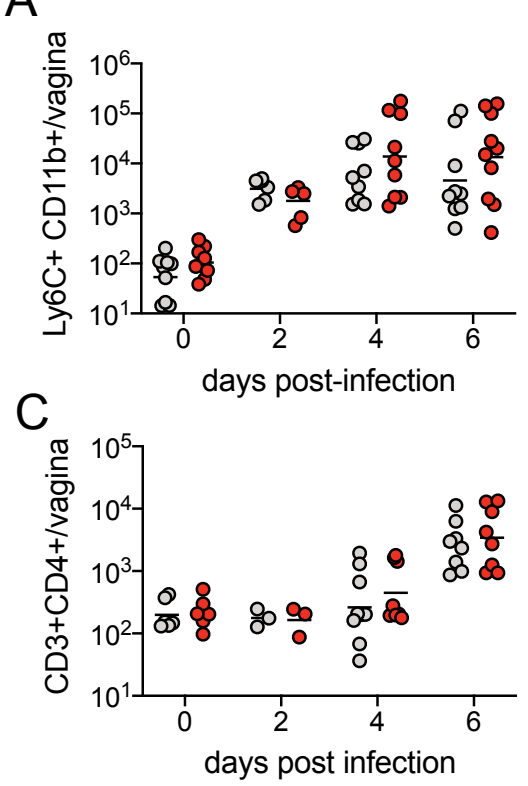

B

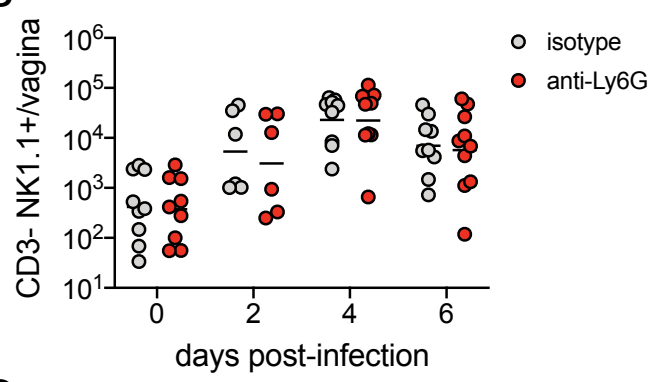

D

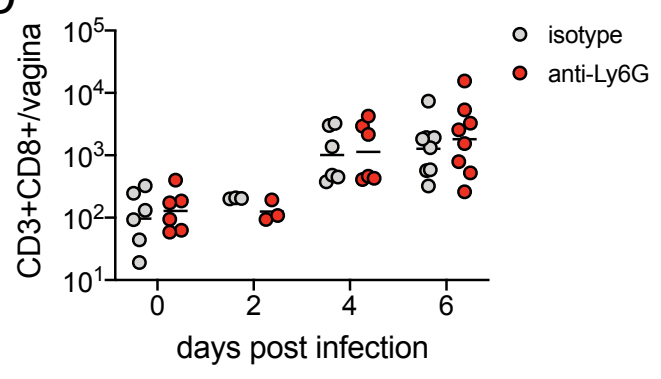

Figure 1 - Supplement 1. Neutrophil depletion does not affect magnitude of the immune cell response after HSV-2 infection. Mice were infected and treated as described in Figure 2. A-D. On the indicated d.p.i., immune cell infiltrates were measured in the vagina by flow cytometry. Ly6C+CD11b+ monocytes (A), CD3-NK1.1+ NK cells $(\mathbf{B}), \mathrm{CD} 3+\mathrm{CD} 4+\mathrm{T}$ cells $(\mathbf{C})$ and CD3+CD8+ T cells $(\mathbf{D})$ were enumerated in perfused tissue. (A, B) Isotype controls: $n=6-9$, anti-Ly6G: $n=6-10$. (C, D) Isotype controls: $n=3-8$, anti-Ly6G: $n=3-9$. All data are pooled from 2 independent experiments. Horizontal bars show mean (A-D). Statistical analysis was performed by two-way ANOVA with Bonferroni's multiple comparisons test. All comparisons were not significant. Raw values for each biological replicate, epsilon values and specific $\mathrm{p}$ values are provided in Figure 1 - Supplement 1 source data. 


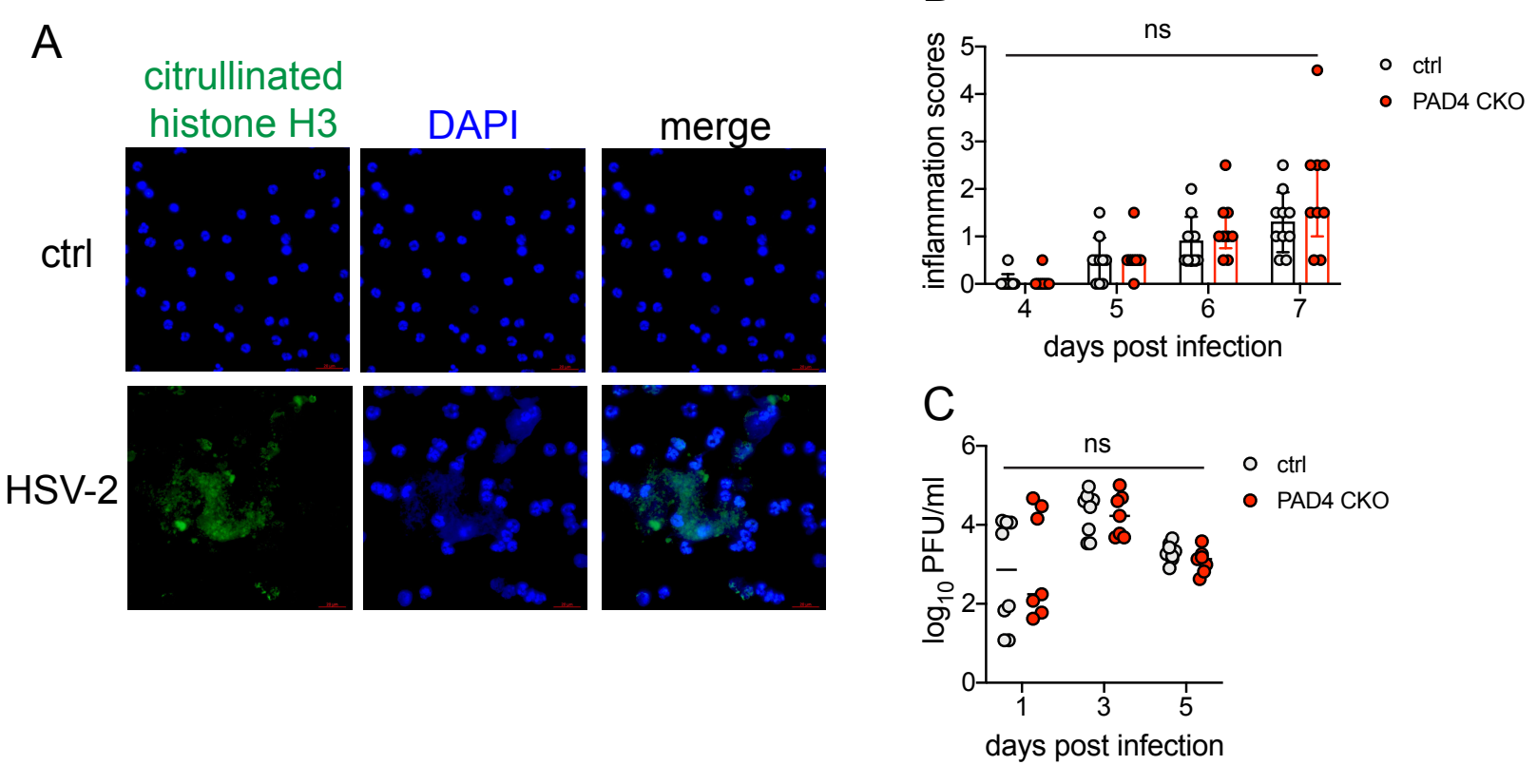

Figure 1 - Supplement 2. PAD4 is not required for development of genital inflammation during HSV-2 infection. A. Neutrophils were isolated from the bone marrow of naive C57BL/6 female mice and stimulated with heat-killed HSV-2 at an MOI of 1 for 4 hours (bottom row) or left unstimulated (top row). NETs are identified by areas of diffuse DAPI staining (blue) that overlap with citrullinated histone H3 (green). Data are representative of 2 independent experiments. B-C. Pad $4{ }^{\mathrm{fl} / \mathrm{fl}} \mathrm{x}$ MRP8-Cre (PAD4 CKO) or Cre- littermate controls were infected with HSV-2 as described in Figure 1. Inflammation scores were monitored for 7 d.p.i. $(\mathrm{Ctrl}: \mathrm{n}=10, \mathrm{KO}: \mathrm{n}=9)(\mathbf{B})$, and infectious virus was measured by plaque assay in vaginal washes collected on the indicated d.p.i. (Ctrl: $n=8, C K O: n=8)(\mathbf{C})$. Data in B-C is pooled from 2 independent experiments. Statistical significance was analyzed by repeated measures two-way ANOVA with $(\mathbf{B})$ or without $(\mathbf{C})$ Geisser-Greenhouse correction and Bonferroni's multiple comparisons test, $\mathrm{ns}=$ not significant. Raw values for each biological replicate, epsilon values and specific $\mathrm{p}$ values are provided in Figure 1 - Supplement 2 source data. 

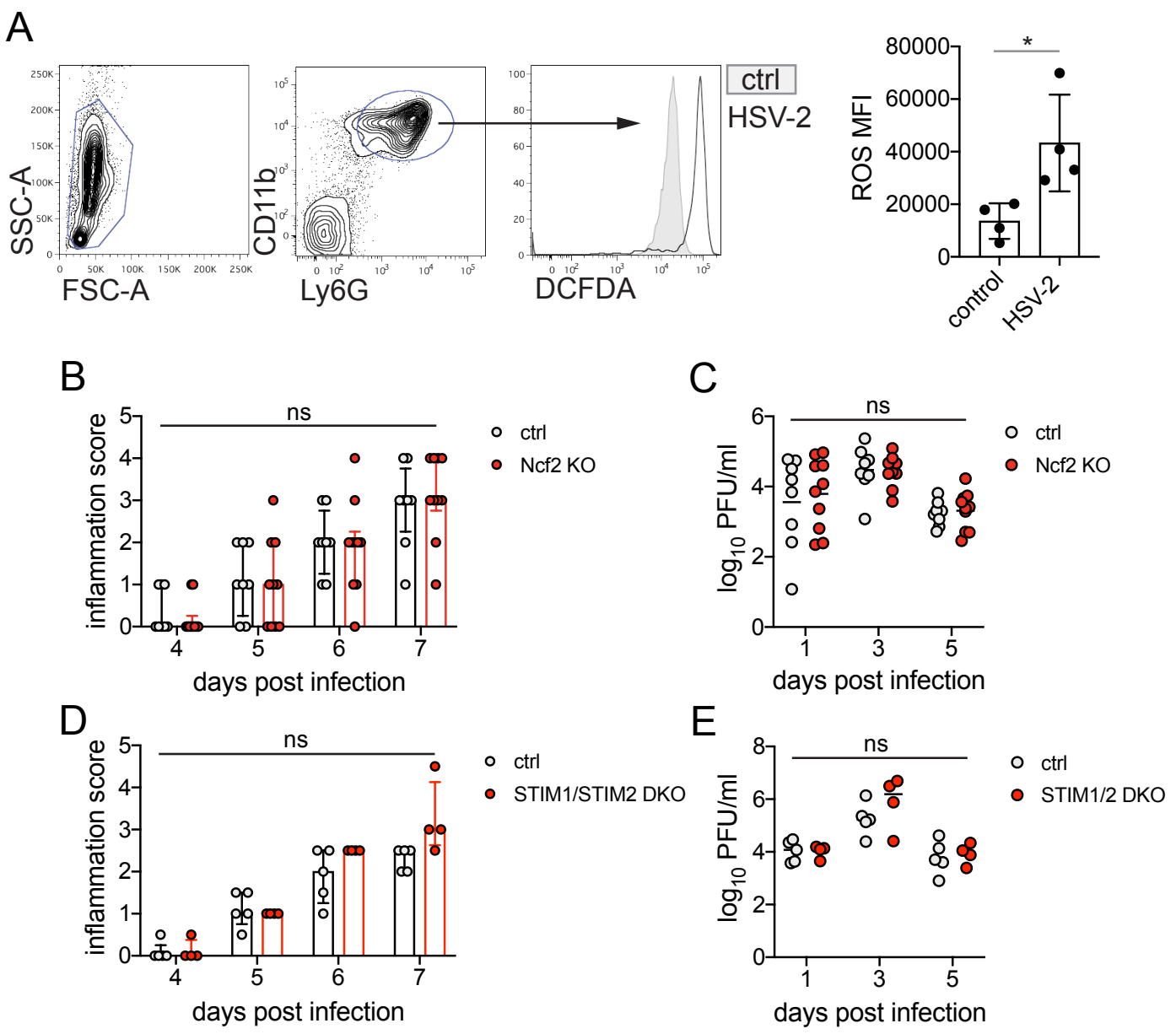

Figure 1 - Supplement 3. ROS production and STIM1/STIM2 expression in neutrophils are not required for genital inflammation after HSV-2 infection. A. Neutrophils were isolated from the bone marrow as using a Histopaque gradient and stimulated with heat-killed HSV-2 at an MOI of 5 for 16 hours $(n=4)$ of left unstimulated $(n=4)$ and then incubated with DCFDA. Plots show gating for leukocytes (left) and CD11b+ Ly6G+ neutrophils (middle). DCFDA fluorescence was measured by flow cytometry in unstimulated (shaded histogram) or HSV-2 stimulated (open histogram) neutrophils. Graph shows mean fluorescence intensity (MFI) of DCFDA (ROS). Ncf2 deficient mice ( $\mathrm{n}=10)$ or littermate controls $(\mathrm{n}=8)(\mathbf{B}-\mathbf{C})$ or STIM1/STIM2 deficient mice (STIM1/STIM2 DKO, $\mathrm{n}=4)$ or littermate controls $(n=5)(\mathbf{D}-\mathbf{E})$ were infected as described in Figure 1. Mice were monitored for genital inflammation for one week after infection (B, D). Infectious virus was measured by plaque assay in vaginal washes collected on the indicated days $(\mathbf{C}, \mathbf{E})$. Bars in A show mean with SD. Bars in $\mathbf{B}$ and $\mathbf{D}$ show median with interquartile range. Bars in $\mathbf{C}$ and $\mathbf{E}$ show mean. Data in A-C are pooled from 2 independent experiments, data in D-E was performed once. Statistical significance was analyzed by unpaired Student's t-test (A), repeated measured two-way ANOVA with $(\mathbf{B}, \mathbf{D})$ or without $(\mathbf{C}, \mathbf{E})$ Geisser-Greenhouse correction and Bonferroni's multiple comparisons test, ${ }^{*} \mathrm{p}<0.05$, ns $=$ not significant. Raw values for each biological replicate, epsilon values and specific $\mathrm{p}$ values are provided in Figure 1 - Supplement 3 source data. 


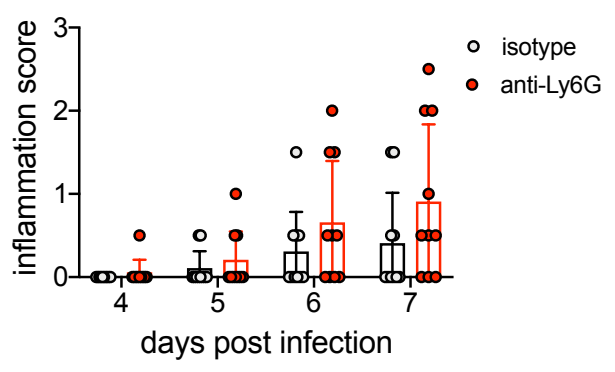

Figure 2 - Supplement 1. Neutrophil depletion prior to HSV-1 genital infection has little impact on disease progression. C57BL/6 J females were infected with $10^{4} \mathrm{PFU}$ HSV-1 McKrae and treated with neutrophil-depleting antibodies as described in Figure 1. Disease progression was measured during the first 7 d.p.i. for anti-Ly6G $(n=10)$ and isotype control $(n=10)$ mice. Bars show median and interquartile range. Data are pooled from 2 independent experiments. Statistical significance was measured by repeated measured two-way ANOVA with Geisser-Greenhouse correction and Bonferroni's multiple comparisons test. All comparisons were not significant. Raw values for each biological replicate, epsilon values and specific $\mathrm{p}$ values are provided in Figure 2 - Supplement 1 source data. 
A

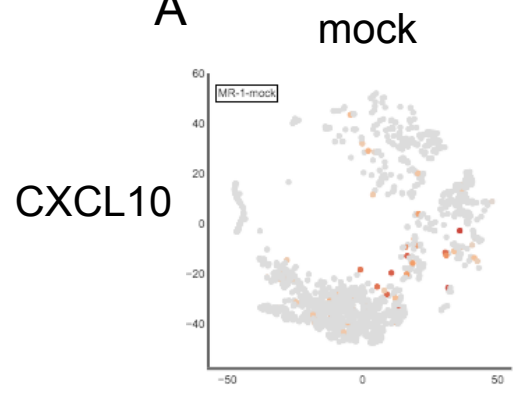

C

Gbp2

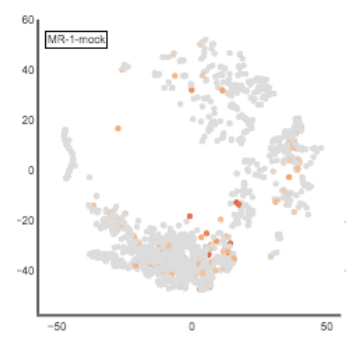

$\mathrm{E}$

$\mathrm{IL}-15$

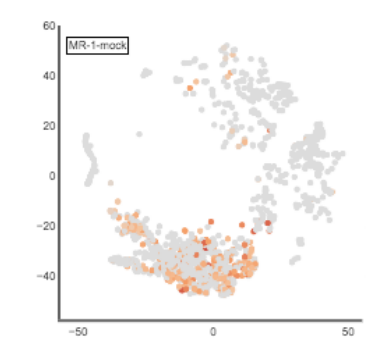

HSV-1
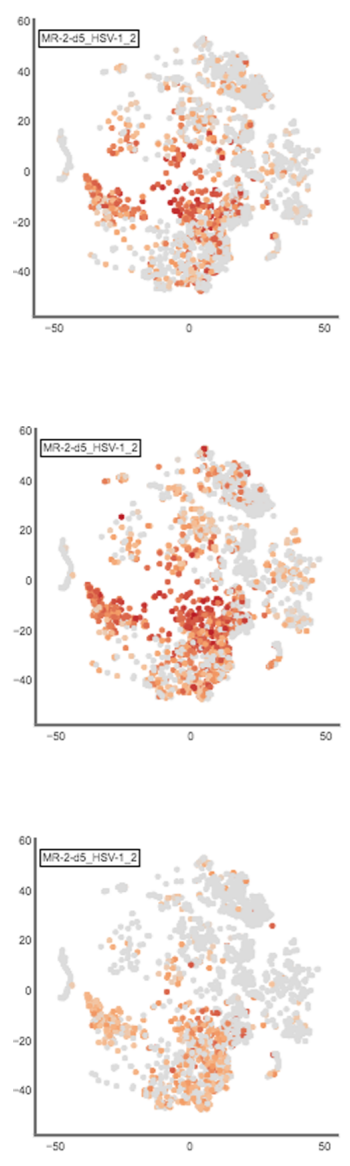

HSV-2
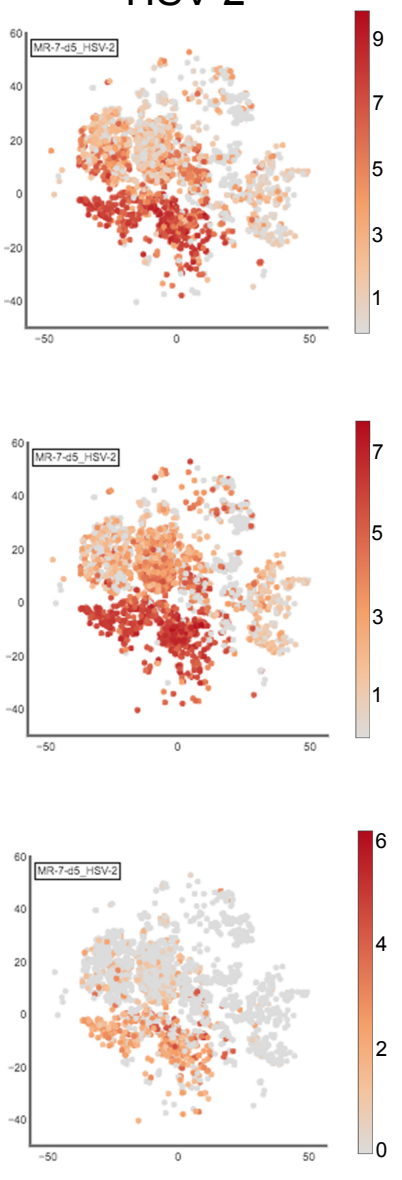

B

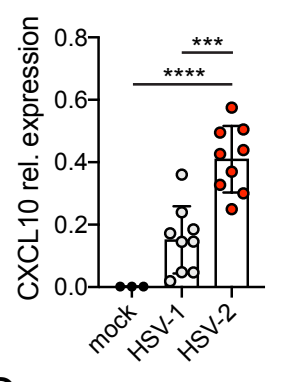

D

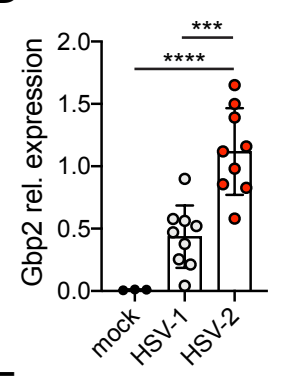

F

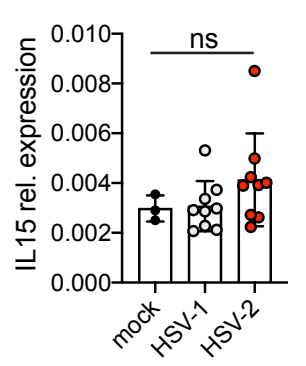

Figure 3 - Supplement 1. Validation of ISG expression in the vagina. Expression of CXCL10 (A, B), Gbp2 (C, D) or IL-15 (E, F) in the vagina of mock inoculated mice or mice at 5 d.p.i. with HSV-1 or HSV-2. tSNE visualization of select ISG transcripts in live vaginal cells profiled by single cell RNA-seq (A, C, E). Expression of the same ISGs relative to Rpl13 was measured by qRT-PCR in whole vaginal tissue harvested from mock inoculated mice $(n=3)$ or mice at 5 d.p.i. with HSV-1 $(n=9)$ or HSV-2 (n=9) (B, D, F). Bars in B, D and F show mean and SD. Data are pooled from 2 independent experiments. Statistical significance was measured by one-way ANOVA with Tukey's multiple comparisons test. $* * * p<0.005, * * * * p<0.001$, ns $=$ not significant. Raw values for each biological replicate and specific p values are provided in Figure 3 - Supplement 1 source data. 


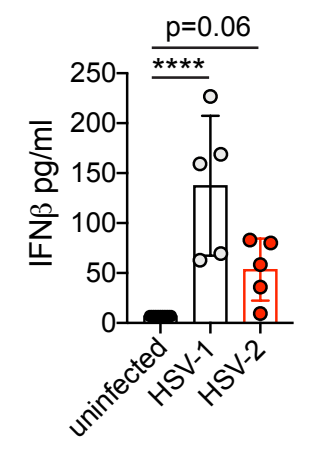

Figure 3 - Supplement 2. Type I IFN is robustly produced in the vagina early after acute HSV-1 or HSV-2 infection. C57BL/6J mice were infected as described in Figure 2. Type I IFN was measured by ELISA in vaginal washes collected from uninfected mice $(n=10)$, or mice at 2 d.p.i. with HSV-1 $(n=5)$ or HSV-2 ( $\mathrm{n}=5$ ). Bars show mean with SD. Experiment was performed once. Statistical significance was measured by one-way ANOVA with Dunnett's multiple comparisons test. ${ }^{* * * *} p<0.001$. Raw values for each biological replicate, epsilon values and specific $\mathrm{p}$ values are provided in Figure 3 - Supplement 2 source data. 

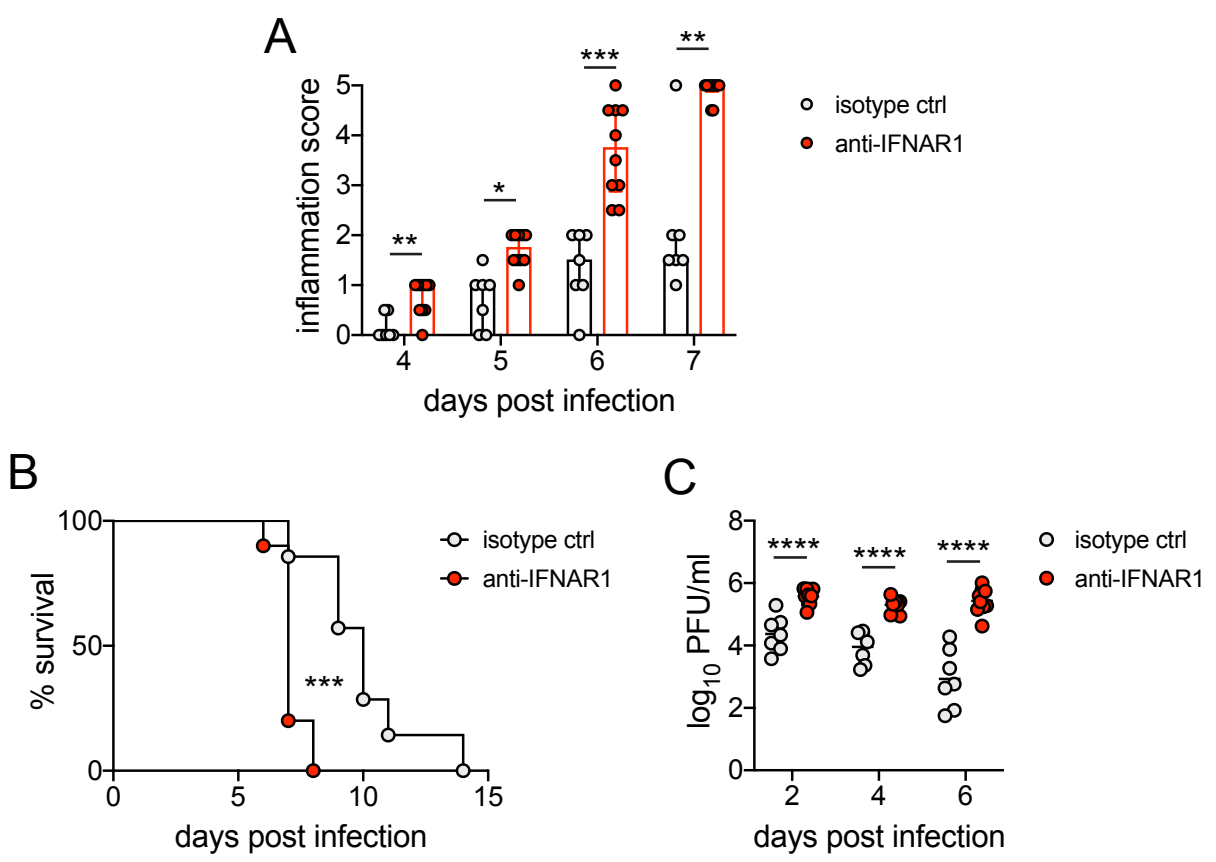

Figure 4 - Supplement 1. Early blockade of IFNAR1 leads to accelerated and more severe disease after HSV-2 infection. C57BL/6J mice were infected as described in Figure 1 . On the day of inoculation, mice were injected i.p. with $1 \mathrm{mg}$ anti-IFNAR1 antibody $(\mathrm{n}=10)$ or an isotype control $(\mathrm{n}=7)$. A. Inflammation scores of anti-IFNAR antibody or isotype control treated mice for the first 7 d.p.i.. B. Survival of mice over the course of two weeks. C. Infectious virus as measured by plaque assay in vaginal washes collected at the indicated d.p.i.. Bars in A show median and interquartile range, bars in $\mathbf{C}$ show mean. Data are pooled from 3 independent experiments. Statistical significance was measured by repeated measured two-way ANOVA with Geisser-Greenhouse correction and Bonferroni's multiple comparisons test (A), log-rank test (B) and mixed-effects analysis with Bonferroni's multiple comparisons test (C). ${ }^{*} \mathrm{p}<0.05, * * \mathrm{p}<0.01, * * * \mathrm{p}<0.005, * * * * \mathrm{p}<0.001$. Raw values for each biological replicate, epsilon values and specific $\mathrm{p}$ values are provided in Figure 4 - Supplement 1 source data. 

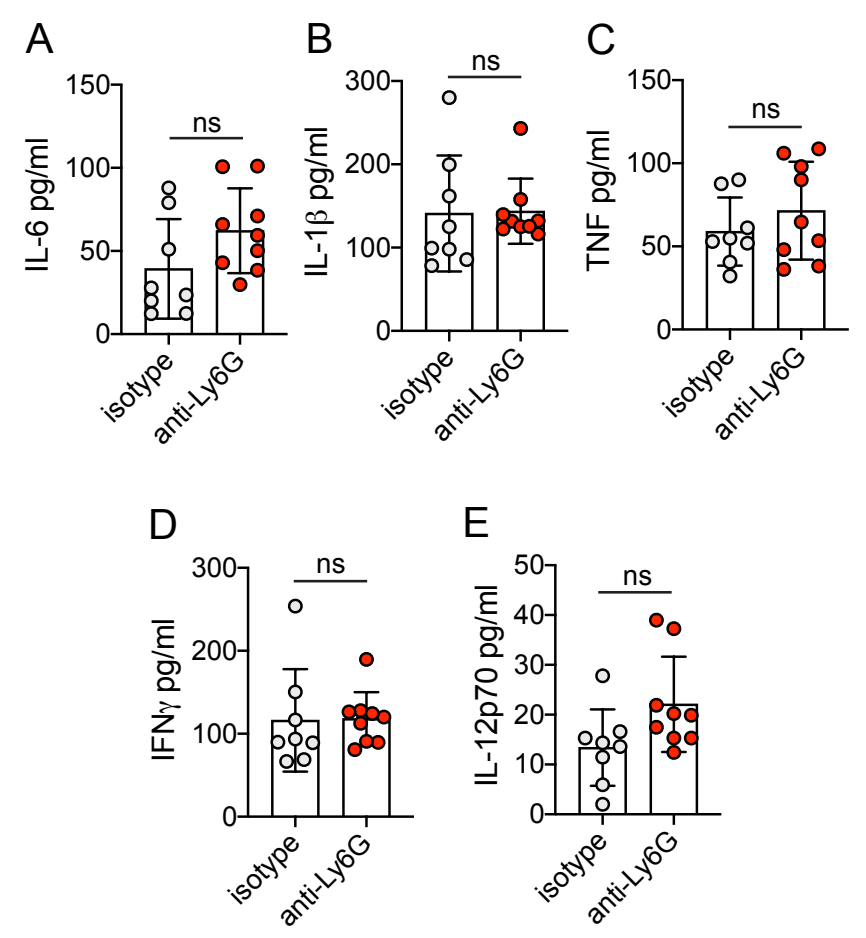

Figure 6 - Supplement 1. Neutrophils do not control production of common pro-inflammatory and antiviral cytokines during HSV-2 infection. Vaginal washes were collected at 5 d.p.i. from mice that were infected and treated as described in Figure 1. IL-6 (A), IL-1b (B), TNF (C), IFN $\gamma$ (D) and IL-12p70 (E) were measured by multiplexed Bioplex assay. Isotype controls: $n=8$, anti-Ly6G: $n=9$. All data are pooled from 2 independent experiments. Statistical analysis was performed by unpaired t-test. $\mathrm{ns}=$ not significant. Raw values for each biological replicate and specific $\mathrm{p}$ values are provided in Figure 6 - Supplement 1 source data. 